\title{
Time-periodic forcing and asymptotic stability for the Navier-Stokes-Maxwell equations
}

\author{
Slim Ibrahim*, Nader Masmoudi† and Pierre Gilles Lemarié-Rieusset ${ }^{\ddagger}$
}

\begin{abstract}
For the 3D Navier-Stokes-Maxwell problem on the whole space and in the presence of external time-periodic forces, first we study the existence of time-periodic small solutions, and then we prove their asymptotic stability. We use new type of spaces to account for averaged decay in time.
\end{abstract}

Keywords: Navier-Stokes equations, periodic solutions, Maxwell's system, dyadic decomposition, maximal regularity, nonlinear estimates 2010 Mathematics Subject Classification: 35B10, 35Q30, 76D05

\section{Introduction.}

Physical and analytical models already exist for both electro-hydrodynamic and magneto-hydrodynamic. However quite often in actual situations, both combined electro and magneto-hydrodynamic effects occur. Recent works attempted to develop fully consistent, multi-dimensional, unsteady and incompressible flows of electrically conducting fluids under the simultaneous or separate influence of externally applied or internally generated electric and magnetic fields. The approach is based on the use of fundamental laws of continuum mechanics and thermodynamics. See for example 12. However, because of the considerable complexity of even simpler versions of the combined electro-magneto-hydrodynamic models, it is still hard to analyze, even numerically, the combined influence of electric and magnetic fields and the fluid flow. In this paper, we analyze an adiabatic situation where thermodynamical effects are neglected, and nonlinearities are reduced to the only action of Lorentz force.

More specifically, consider a three-dimensional incompressible, viscous and

\footnotetext{
${ }^{*}$ Department of Mathematics and Statistics, University of Victoria; e-mail: ibrahims@uvic.ca

${ }^{\dagger}$ Courant Institute, New-York University; e-mail: masmoudi@courant.nyu.edu

${ }^{\ddagger}$ Laboratoire de Mathématiques et Modélisation d'Évry, UMR CNRS 9071, UEVE, ENSIIE; e-mail: plemarie@univ-evry.fr
} 
charged fluid with a velocity field $\vec{u}$. Fluid charged particles motion generates an electro-magnetic field $(\vec{E}, \vec{B})$ satisfying Maxwell equations, and a current $\vec{J}$ that acts backs on the fluid through Lorentz force. We assume that the current is given by Ohm's law $J=\sigma(\vec{E}+\vec{u} \wedge \vec{B})$. Thus, the Navier-Stokes-Maxwell system we study reads as

$$
\left\{\begin{aligned}
\partial_{t} \vec{u}+\operatorname{div}(\vec{u} \otimes \vec{u}) & =\nu \Delta \vec{u}+\vec{J} \wedge \vec{B}+\vec{F}_{\mathrm{per}}-\vec{\nabla} p \\
\partial_{t} \vec{E}-\vec{\nabla} \wedge \vec{B} & =-\vec{J}+\vec{G}_{\mathrm{per}} \\
\partial_{t} \vec{B}+\vec{\nabla} \wedge \vec{E} & =\vec{H}_{\mathrm{per}} \\
\operatorname{div} \vec{u}=\operatorname{div} \vec{B} & =0 \\
\vec{J} & =\sigma(\vec{E}+\vec{u} \wedge \vec{B}) .
\end{aligned}\right.
$$

Here, $\vec{u}, \vec{E}, \vec{B}: \mathbb{R}_{t}^{+} \times \mathbb{R}_{x}^{3} \longrightarrow \mathbb{R}^{3}$ are vector fields defined on $\mathbb{R}^{3}$, and the scalar function $p$ stands for the pressure. The positive parameters $\nu$ and $\sigma$ represent the viscosity of the fluid and the electric resistivity, respectively. The self-interaction force term $\vec{J} \times \vec{B}$ in the Navier-Stokes equations comes from the Lorentz force under a quasi-neutrality assumption of the net charge carried by the fluid. Notice that taking into account a moving reference frame of the fluid, yields the correction $u \times B$ to the classical Ohm's law and keeps Faraday's law invariant under Gallilean transformation. The second equation in (0.1) is the Ampère-Maxwell equation for the electric field $\vec{E}$. The third equation is nothing but Faraday's law. For a detailed introduction to similar models and the theory of MHD, we refer to Davidson [9] and Biskamp [3]. In (0.1), the external forces $\vec{F}_{\text {per }}, \vec{G}_{\text {per }}$ and $\vec{H}_{\text {per }}$ are taken time-periodic: for a fixed time period $T>0$, we have

$\vec{F}_{\text {per }}(t+T, x)=\vec{F}_{\text {per }}(t, x), \vec{G}_{\text {per }}(t+T, x)=\vec{G}_{\text {per }}(t, x), \vec{H}_{\text {per }}(t+T, x)=\vec{H}_{\text {per }}(t, x)$.

Before going any further, let us emphasize that despite the possible nonphysical full relevance of (10.1), the system still captures the various mathematical challenges of the full complicated original set of equations. Indeed, (0.1) is a coupling of a dissipative equation of parabolic type (Navier-Stokes) with a hyperbolic system (Maxwell's equations). Despite the presence of damping in Ohm's law, solutions to Maxwell's equations do not enjoy any smoothing effect, due to the hyperbolic nature of the equations. Moreover, as this will be seen, a few challenges also arise when dealing with different decay rates, for different linear parts, and also different frequency sizes caused by the coupling.

Note that the pressure $p$ can still be determined using Leray projection from $\vec{u}$ and $\vec{J} \wedge \vec{B}$ via an explicit Caldéron-Zygmund operator (see [6] for instance):

$$
\vec{\nabla} p=\vec{\nabla} \frac{1}{\Delta} \operatorname{div}\left(\vec{J} \wedge \vec{B}+\vec{F}_{\text {per }}-\operatorname{div}(\vec{u} \otimes \vec{u})\right)
$$


In all what follows, we denote the solution to (0.1) by

$$
\vec{\Gamma}:=(\vec{U}, \vec{E}, \vec{B}) .
$$

When no exterior forces act on the system (0.1), the initial value problem reads

$$
\left\{\begin{aligned}
& \partial_{t} \vec{u}+\operatorname{div}(\vec{u} \otimes \vec{u})=\Delta \vec{u}+\vec{J} \wedge \vec{B}-\vec{\nabla} p \\
& \partial_{t} \vec{E}-\vec{\nabla} \wedge \vec{B}=-\vec{J} \\
& \partial_{t} \vec{B}+\vec{\nabla} \wedge \vec{E}=0 \\
& \operatorname{div} \vec{u}=\operatorname{div} \vec{B}=0 \\
& \vec{J}=\vec{E}+\vec{u} \wedge \vec{B} \\
& \vec{u}(0, .)=\vec{u}_{0}, \quad \vec{E}(0, .)=\vec{E}_{0}, \quad \vec{B}(0, .)=\vec{B}_{0} .
\end{aligned}\right.
$$

Solutions to (0.2) formally enjoy the energy balance

$$
\frac{1}{2} \frac{d}{d t}\left[\|\vec{u}\|_{L^{2}}^{2}+\|\vec{B}\|_{L^{2}}^{2}+\|\vec{E}\|_{L^{2}}^{2}\right]+\|\vec{J}\|_{L^{2}}^{2}+\|\nabla \vec{u}\|_{L^{2}}^{2}=0,
$$

which suggests that weak solutions would exist in the space

$$
\vec{u} \in L^{\infty}\left(0, \infty ; L^{2}\right) \cap L^{2}\left(0, \infty ; \dot{H}^{1}\right) ; \quad \vec{E}, \vec{B} \in L^{\infty}\left(0, \infty ; L^{2}\right) \quad \vec{J} \in L^{2}\left(0, \infty ; L^{2}\right) .
$$

Unfortunately, weak solutions are not known to exist even in two space dimension. In [18, Masmoudi proved the existence of global strong solutions. Later on, Ibrahim and Keraani [15] relaxed the regularity condition on the initial data to construct global small solutions à la Kato. This result was recently improved by Germain, Ibrahim and Masmoudi [11] by taking small initial data $\vec{u}_{0}, \vec{E}_{0}$ and $\vec{B}_{0}$ in $\dot{H}^{1 / 2}$, and construct a solution $(\vec{u}, \vec{E}, \vec{B})$ such that $\vec{u} \in L^{\infty} \dot{H}^{1 / 2} \cap L^{2} \dot{H}^{3 / 2} \cap L^{2} L^{\infty}, \vec{E} \in L^{\infty} \dot{H}^{1 / 2} \cap L^{2} \dot{H}^{1 / 2}$ and $\vec{B} \in L^{\infty} \dot{H}^{1 / 2}$.

This paper is organized as follows. In section one, we introduce some useful notation and state our results: A first Theorem about the existence of time-periodic solutions in spaces of Sobolev type with an extra spatial regularity. Then, we relax the hypothesis of the first Theorem and extend it to critical Besov type spaces. A such an extension seems to us necessary in order to prove the last result about the stability of the periodic-in time solutions. Section two is devoted to the proof of the two existence results, and we only give the full details in the case of Sobolev. In section three, we start by examining a maximal regularity result adapted to the spaces that incorporates averaged decay in time. Then, we show the decay of the electromagnetic field where we use the full spectral properties of the weakly damped Maxwell's equations. Then, we list all the nonlinear estimates that appear in the study of the nonlinear stability, and we only prove the worst two of them when two factors have no decay in time. The manuscript is 
then finished with an Appendix summarizing the main spectral properties of the weakly damped Maxwell's equations.

\section{Acknowledgements}

Slim Ibrahim is partially supported by NSERC Discovery grant \# 3716372014. Nader Masmoudi is in part supported by NSF grant DMS-1211806\# Slim Ibrahim would like to thank the University of Évry and the University of Paris Diderot-Paris 7 for their hospitality to accomplish a part of this work.

\section{Notation}

The well-known Littlewood-Paley decomposition and the corresponding frequency cut-off operators will be of frequent use in this paper. We briefly recall it to define the functional spaces we need.

There exists a radial positive function $\varphi \in \mathcal{D}\left(\mathbb{R}^{d} \backslash\{0\}\right)$ such that

$$
\begin{aligned}
\sum_{q \in \mathbb{Z}} \varphi\left(2^{-q} \xi\right) & =1 \quad \forall \xi \in \mathbb{R}^{d} \backslash\{0\}, \\
\operatorname{Supp} \varphi\left(2^{-\mathrm{q}} \cdot\right) \cap \operatorname{Supp} \varphi\left(2^{-\mathrm{j}} \cdot\right) & =\emptyset, \quad \forall|q-j| \geq 2 .
\end{aligned}
$$

For every $q \in \mathbb{Z}$ and $v \in \mathcal{S}^{\prime}\left(\mathbb{R}^{d}\right)$ we set

$$
\Delta_{q} v=\mathcal{F}^{-1}\left[\varphi\left(2^{-q} \xi\right) \hat{v}(\xi)\right] \quad \text { and } S_{q}=\sum_{j=-\infty}^{q-1} \Delta_{j} .
$$

Bony's decomposition [4] consists in splitting the product uv into three part:1:

$$
u v=T_{u} v+T_{v} u+R(u, v),
$$

with

$$
T_{u} v=\sum_{q} S_{q-1} u \Delta_{q} v, \quad R(u, v)=\sum_{q} \Delta_{q} u \tilde{\Delta}_{q} v \quad \text { and } \quad \tilde{\Delta}_{q}=\sum_{i=-1}^{1} \Delta_{q+i} .
$$

For $(p, r) \in[1,+\infty]^{2}$ and $s \in \mathbb{R}$ we define the homogeneous Besov space $\dot{B}_{p, r}^{s}$ as the set of $u \in \mathcal{S}^{\prime}\left(\mathbb{R}^{d}\right)$ such that $u=\sum_{q} \Delta_{q} u$ and

$$
\|u\|_{\dot{B}_{p, r}^{s}}=\left\|\left(2^{q s}\left\|\Delta_{q} u\right\|_{L^{p}}\right)_{q \in \mathbb{Z}}\right\|_{\ell^{r}(\mathbb{Z})}<\infty
$$

\footnotetext{
${ }^{1}$ It should be said that this decomposition is true in the class of distributions for which $\sum_{q \in \mathbb{Z}} \Delta_{q}=I$. For example, polynomial functions do not belong to this class.
} 
In the case $p=r=2$, the space $\dot{B}_{2,2}^{s}$ turns out to be the classical homogeneous Sobolev space $\dot{H}^{s}$.

In order to prove the our stability result, we need to build spaces that take into account the different decay rates coming from the coupling of the two types of PDEs (Navier-Stokes, and Maxwell), and also the weak decay of the low frequencies in the Maxwell's equations. In addition, and in order to estimate the nonlinear terms, we need to introduce spaces that capture an average decay in time, and not just pointwise decay. This will be crucial in our analysis. We define the spaces that distinguish between the high and low frequencies of a function

Definition 1.1. Let $\Delta_{q}$ denote the dyadic frequency localization operator defined in section 1. We define a space that distinguishes between the high and low frequencies of a function as follows. For $s_{1}, s_{2} \in \mathbb{R}$ and $1 \leq p, q_{1}, q_{2} \leq \infty$ define the space $\dot{\mathcal{B}}_{p, q}^{s_{1}, s_{2}}$ by its norm

$$
\|\phi\|_{\dot{\mathcal{B}}_{p,\left(q_{1}, q_{2}\right)}^{\left(s_{1}, s_{2}\right)}}:=\left(\sum_{k \leq 0} 2^{k q s_{1}}\left\|\Delta_{k} \phi\right\|_{L^{p}}^{q_{1}}\right)^{\frac{1}{q_{1}}}+\left(\sum_{k>0} 2^{k q s_{2}}\left\|\Delta_{k} \phi\right\|_{L^{p}}^{q_{2}}\right)^{\frac{1}{q_{2}}} .
$$

We will also use the short-hands

$$
\text { and } \quad \dot{B}_{p,\left(q_{1}, q_{2}\right)}^{s}:=\dot{\mathcal{B}}_{p,\left(q_{1}, q_{2}\right)}^{(s, s)}, \quad \dot{H}^{s}=\dot{\mathcal{B}}_{2,(2,2)}^{(s, s)}, \quad \text { and } \quad \dot{H}^{s, t}:=\dot{\mathcal{B}}_{2,(2,2)}^{(s, t)} .
$$

Finally, define the space-time functional space $\tilde{L}_{T}^{r} \dot{\mathcal{B}}_{p,\left(q_{1}, q_{2}\right)}^{\left(s_{1}, s_{2}\right)}$ by its norm

$$
\|\phi\|_{\tilde{L}_{T}^{r} \dot{\mathcal{B}}_{p,\left(q_{1}, q_{2}\right)}^{\left(s_{1}, s_{2}\right)}}:=\left(\sum_{k \leq 0} 2^{q_{1} s_{1} k}\left\|\Delta_{k} \phi\right\|_{L_{T}^{r} L^{p}}^{q_{1}}\right)^{\frac{1}{q_{1}}}+\left(\sum_{k>0} 2^{q_{2} k s_{2}}\left\|\Delta_{k} \phi\right\|_{L_{T}^{r} L^{p}}^{q_{2}}\right)^{\frac{1}{q_{2}}},
$$

with the trivial extension when $r=\infty$. We also define the new spaces that take into account an averaged decay in time. Precisely, we denote

$$
\begin{aligned}
\operatorname{sũp}_{n \in \mathbb{N}}(n+1)^{\frac{1-\varepsilon}{2}}\|u\|_{L^{2}(n, n+1) ; \dot{\mathcal{B}}_{p,\left(q_{1}, q_{2}\right)}^{\left(s_{1}, s_{2}\right)}}: & =\left(\sum_{k \leq 0} 2^{q_{1} s_{1} k} \sup _{n \in \mathbb{N}}(n+1)^{\frac{(1-\varepsilon) q_{1}}{2}}\left\|\Delta_{k} u\right\|_{L^{2}\left(n, n+1 ; L^{p}\right)}^{q_{1}}\right)^{\frac{1}{q_{1}}} \\
& +\left(\sum_{k \leq 0} 2^{q_{2} s_{2} k} \sup _{n \in \mathbb{N}}(n+1)^{\frac{(1-\varepsilon) q_{2}}{2}}\left\|\Delta_{k} u\right\|_{L^{2}\left(n, n+1 ; L^{p}\right)}^{q_{2}}\right)^{\frac{1}{q_{2}}} .
\end{aligned}
$$

with the obvious generalizations in the cases $q_{j}=\infty$, or $\tilde{L}_{T}^{r} \dot{H}^{s}$ etc..

The space $\dot{\mathcal{B}}_{p,\left(q_{1}, q_{2}\right)}^{\left(s_{1}, s_{2}\right)}$ is nothing but the usual Besov space $\dot{\mathcal{B}}_{p, q_{2}}^{s_{2}}$ for high frequencies while it behaves like $\dot{\mathcal{B}}_{p, q_{1}}^{s_{1}}$ for low frequencies. If $s_{1}>s_{2}$, it is not difficult to see that $\dot{\mathcal{B}}_{p,\left(q_{1}, q_{2}\right)}^{\left(s_{1}, s_{2}\right)}=\dot{\mathcal{B}}_{p, q_{1}}^{s_{1}}+\dot{\mathcal{B}}_{p, q_{2}}^{s_{2}}$. The $\tilde{L}$ type spaces were first used by Chemin and Lerner [8].

In the sequel, consider a parameter $0<\varepsilon<1$, introduce the spaces $\mathcal{X}_{1}$, $\mathcal{X}_{2}, \mathcal{X}_{3}$, and their "dual" counterparts $\mathcal{Y}_{1}$ and $\mathcal{Y}_{2}$ by defining their norms.

$$
\|u\|_{\mathcal{X}_{1}}:=\operatorname{supp}_{t>0}(t+1)^{\frac{1-\varepsilon}{2}}\|u(t)\|_{\dot{\mathcal{B}}_{2,(\infty, 1)}^{\left(\frac{3}{2}-\varepsilon, \frac{1}{2}\right)}}+\operatorname{sunp}_{n \in \mathbb{N}}(n+1)^{\frac{1-\varepsilon}{2}}\|u\|_{L^{2}\left(n, n+1 ; \dot{\mathcal{B}}_{2,(\infty, 1)}^{\frac{3}{2}}\right)}
$$




$$
\begin{aligned}
& \|E\|_{\mathcal{X}_{2}}:=\operatorname{supp}_{t>0}(t+1)^{\frac{1-\varepsilon}{2}}\|E(t)\|_{H^{\frac{1}{2}}} \sim \operatorname{sunp}_{n \in \mathbb{N}}(n+1)^{\frac{1-\varepsilon}{2}}\|E\|_{L^{\infty}\left(n, n+1 ; H^{\frac{1}{2}}\right)} \\
& \|B\|_{\mathcal{X}_{3}}:=\operatorname{supp}_{t>0}(t+1)^{\frac{1-\varepsilon}{2}}\|B(t)\|_{\dot{H}^{1, \frac{1}{2}}} \sim \sup _{n \in \mathbb{N}}(n+1)^{\frac{1-\varepsilon}{2}}\|B\|_{L^{\infty}\left(n, n+1 ; \dot{H}^{1, \frac{1}{2}}\right)}, \\
& \|F\|_{\mathcal{Y}_{1}}:=\operatorname{supp}_{n \in \mathbb{N}}(n+1)^{\frac{1-\varepsilon}{2}}\|F\|_{L^{2}\left(n, n+1 ; \dot{\mathcal{B}}_{2,(\infty, 1)}^{\left(-\frac{1}{2}-\varepsilon,-\frac{1}{2}\right)}\right)}, \\
& \|G\|_{\mathcal{Y}_{2}}:=\operatorname{sũp}_{n \in \mathbb{N}}(n+1)^{\frac{1-\varepsilon}{2}}\|G\|_{L^{2}\left(n, n+1 ; H^{\frac{1}{2}}\right)}
\end{aligned}
$$

Finally, let $\mathcal{X}:=\left(\mathcal{X}_{1} \cap \tilde{L}^{\infty}\left(\dot{\mathcal{B}}_{2,(\infty, 1)}^{\frac{1}{2}}\right) \times \mathcal{X}_{2} \times \mathcal{X}_{3}\right.$.

\section{$1.1 \quad$ Results}

In our first result and under a smallness assumption on the forces, we construct (in Sobolev spaces with an extra $\delta$ regularity) a time-periodic solution $\vec{\Gamma}_{\text {per }}$ to (0.1). More precisely, we have

\section{Theorem 1.1.}

Let $0<\delta<1$. Then there exists a positive constant $\epsilon_{T, \delta}$ such that : if the time-periodic forces $\vec{F}_{\mathrm{per}}, \vec{G}_{\mathrm{per}}$ and $\vec{H}_{\mathrm{per}}$ satisfy the following assumptions :

1. $\vec{F}_{\mathrm{per}}$ belongs to $L_{\mathrm{per}}^{2} \dot{H}^{-\frac{1}{2}} \cap L_{\mathrm{per}}^{2} \dot{H}^{-\frac{1}{2}+\delta}$ and

$$
\sqrt{\int_{0}^{T}\left\|\vec{F}_{\mathrm{per}}(t, .)\right\|_{\dot{H}^{-\frac{1}{2}}}^{2} d t}+\sqrt{\int_{0}^{T}\left\|\vec{F}_{\mathrm{per}}(t, .)\right\|_{\dot{H}^{-\frac{1}{2}+\delta}}^{2} d t}<\epsilon_{T, \delta}
$$

2. the mean value $\vec{F}_{0}=\frac{1}{T} \int_{0}^{T} \vec{F}_{\mathrm{per}}(t,) d$.$t belongs to \dot{B}_{2, \infty}^{-\frac{3}{2}}$ and

$$
\left\|\vec{F}_{0}\right\|_{\dot{B}_{2, \infty}^{-3 / 2}}<\epsilon_{T, \delta}
$$

3. $\vec{G}_{\mathrm{per}}$ belongs to $L_{\mathrm{per}}^{2} H^{\frac{1}{2}+\delta}$ and

$$
\sqrt{\int_{0}^{T}\left\|\vec{G}_{\mathrm{per}}(t, .)\right\|_{H^{\frac{1}{2}+\delta}}^{2} d t}<\epsilon_{T, \delta}
$$

4. the mean value $\vec{G}_{0}=\frac{1}{T} \int_{0}^{T} \vec{G}_{\mathrm{per}}(t,) d$.$t belongs to \dot{H}^{-1}$ and

$$
\left\|\vec{G}_{0}\right\|_{\dot{H}^{-1}}<\epsilon_{T, \delta},
$$


5. $\vec{H}_{\mathrm{per}}$ is divergence-free (div $\vec{H}_{\mathrm{per}}=0$ ) and $\vec{H}_{\mathrm{per}}$ belongs to $L_{\mathrm{per}}^{2} H^{\frac{1}{2}+\delta}$ with

$$
\sqrt{\int_{0}^{T}\left\|\vec{H}_{\mathrm{per}}(t, .)\right\|_{H^{\frac{1}{2}+\delta}}^{2} d t}<\epsilon_{T, \delta}
$$

6. the mean value $\vec{H}_{0}=\frac{1}{T} \int_{0}^{T} \vec{H}_{\text {per }}(t,) d$.$t belongs to \dot{H}^{-2}$ and

$$
\left\|\vec{H}_{0}\right\|_{\dot{H}^{-2}}<\epsilon_{T, \delta},
$$

then the Navier-Stokes-Maxwell problem (0.1) has a time-periodic solution $\left(\vec{u}_{\text {per }}, \vec{E}_{\text {per }}, \vec{B}_{\text {per }}\right)$ such that :

- $\vec{u}_{\mathrm{per}}$ belongs to $L_{\mathrm{per}}^{\infty} \dot{B}_{2, \infty}^{\frac{1}{2}} \cap L_{\mathrm{per}}^{2} \dot{H}^{\frac{3}{2}+\delta}$

- $\vec{E}_{\mathrm{per}}$ and $\vec{B}_{\mathrm{per}}$ belong to $L_{\mathrm{per}}^{\infty} H^{\frac{1}{2}+\delta}$.

We extend the above statement to solutions in critical spaces of Besovtype. This will be crucial for the stability as we were not able to prove the stability in the spaces given by Theorem 1.1. More precisely, we have

\section{Theorem 1.2.}

Let $T>0$ denote the time period of three periodic forces $\vec{F}_{p e r}, \vec{G}_{p e r}$ and $\vec{H}_{\text {per }}$ decomposed as follows into a fluctuating and zero mean parts:

$\vec{F}_{p e r}(t, x):=\vec{F}_{0}(x)+\vec{F}_{f}(t, x), \vec{G}_{p e r}:=\vec{G}_{0}(x)+\vec{G}_{f}(t, x)$ and $\vec{H}_{p e r}:=$ $\vec{H}_{0}(x)+\vec{H}_{f}(t, x)$ with

$$
\int_{0}^{T} \vec{F}_{f} d t=\int_{0}^{T} \vec{G}_{f} d t=\int_{0}^{T} \vec{H}_{f} d t=0
$$

There exists $\varepsilon_{T}>0$ such that under the following smallness assumptions

$$
\begin{gathered}
\left\|\vec{F}_{p e r}\right\|_{\tilde{L}^{2}\left(0, T ; \dot{\mathcal{B}}_{2,(\infty, 1)}^{-\frac{1}{2}}\right)}+\left\|F_{0}\right\|_{\dot{\mathcal{B}}_{2,(\infty, 1)}^{-\frac{3}{2}}} \leq \varepsilon_{T} \\
\left\|\vec{G}_{p e r}\right\|_{L^{2}\left(0, T ; H^{\frac{1}{2}}\right)}+\left\|\vec{G}_{0}\right\|_{\dot{H}^{-1}} \leq \varepsilon_{T}
\end{gathered}
$$

and

$$
\left\|\vec{H}_{p e r}\right\|_{L^{2}\left(0, T ; H^{\frac{1}{2}}\right)}+\left\|\vec{H}_{0}\right\|_{\dot{H}^{-2}} \leq \varepsilon_{T},
$$

a unique mild solution $\vec{\Gamma}_{\text {per }}=\left(\vec{u}_{\text {per }}, \vec{E}_{\text {per }}, \vec{B}_{\text {per }}\right)$ of (0.1) exists such that $\vec{u}_{p e r} \in \tilde{L}_{p e r}^{\infty} \dot{\mathcal{B}}_{2,(\infty, 1)}^{\frac{1}{2}} \cap \tilde{L}_{p e r}^{2} \dot{\mathcal{B}}_{2,(\infty, 1)}^{\frac{3}{2}}$ and $\vec{E}_{p e r}, \vec{B}_{p e r} \in \tilde{L}_{p e r}^{\infty} H^{\frac{1}{2}}$.

Another variant of the existence result of time periodic solutions is given by the following theorem where we require a slightly better control of the high frequencies of the solution $\vec{\Gamma}$. 
Remark 1.1. - One can prove local existence if the low frequency part of the initial data of the velocity field is in $\dot{\mathcal{B}}_{2,(\infty, 1)}^{\frac{1}{2}}$ and its high frequency is in $\dot{H}^{\frac{1}{2}}$.

- Compared to the results of [11] in the absence of forcing terms, the statement of Theorem 1.1 requires a slightly better control of high frequencies for $\vec{u}, \vec{E}$ and $\vec{B}$, a better control of low frequencies of $\vec{E}$ and $\vec{B}$, and a weaker control on the low frequencies of $\vec{u}$.

- Our proof also shows that the more regular is the forcing, the more regular will be its corresponding periodic-in time solution. Indeed, if for example the forcing is small in

$$
\vec{F}_{\mathrm{per}} \in L_{\mathrm{per}}^{2} \dot{H}^{-\frac{1}{2}} \cap L_{\mathrm{per}}^{2} \dot{H}^{\frac{1}{2}}, \quad \vec{G}_{\mathrm{per}} \in L_{\mathrm{per}}^{2} H^{\frac{3}{2}}, \quad \vec{H}_{\mathrm{per}} \in L_{\mathrm{per}}^{2} H^{\frac{3}{2}},
$$

then, we have

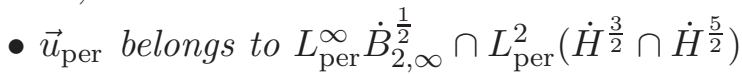

- $\vec{E}_{\mathrm{per}}$ and $\vec{B}_{\text {per }}$ belong to $L_{\mathrm{per}}^{\infty} H^{\frac{3}{2}}$.

Next, we study the stability of the time-periodic solutions: what happens when, at some time $t_{0}$, one takes a perturbation of the solutions constructed in above:

$$
\vec{\Gamma}\left(t_{0}\right)=\vec{\Gamma}_{\text {per }}\left(t_{0}\right)+\vec{\Gamma}_{\text {err }}
$$

with $\vec{\Gamma}_{\text {err }}$ small in $\dot{B}_{2,(\infty, 1)}^{1 / 2} \times H^{1 / 2} \times H^{1 / 2}$ ? Do we have a global solution of (0.1) on $\left[t_{0},+\infty\right)$ and does the error go to 0 in suitable norms when $t$ goes to $+\infty$ ? The main problem rises when we estimate the cross terms coming from the interactions between the periodic solution and the solution we want to construct. The worst interaction is given by a term of the type

$$
\left(\vec{U}_{\text {per }} \wedge \vec{B}_{\text {per }}\right) \wedge \vec{B}
$$

first because of the non-decay of $U_{\text {per }}$, and $B_{\text {per }}$, and second because we barley miss an $L^{\infty}\left(L^{\infty}\right)$ estimate on $U_{\text {per }}$. To overcome such a problem, we impose a strong condition on the low frequencies of the velocity field. In doing so, we are obliged to allow an- $\varepsilon$ loss in the time decay rate. It is important to notice that because of this problem, we were not able to show the stability of the the solutions given by Theorem 1.1. Hence, our second main result is the following.

Theorem 1.3. Given three T-periodic forces $\vec{F}_{\text {per }}(t), \vec{G}_{\text {per }}(t)$ and $\vec{H}_{\text {per }}(t)$ satisfying the hypothesis of Theorem 1.2, and denote by $\vec{\Gamma}_{\text {per }}(t)$ the corresponding small T-periodic solution. Consider an initial data $\vec{\Gamma}_{\text {err }}^{0}+\vec{\Gamma}_{p e r}(0)$ 
with $\vec{\Gamma}_{\text {err }}^{0}$ small in $\dot{\mathcal{B}}_{2,(\infty, 1)}^{\frac{1}{2}} \times H^{\frac{1}{2}} \times H^{\frac{1}{2}}$, there exists $\overrightarrow{\bar{\Gamma}}$ a global solution of (0.1) with that initial data $\vec{\Gamma}_{\text {err }}^{0}+\vec{\Gamma}_{\text {per }}(0)$. Moreover, we have

$$
\overrightarrow{\bar{\Gamma}}-\vec{\Gamma}_{p e r} \in \mathcal{X},
$$

so that $\vec{\Gamma}$ converges asymptotically to $\vec{\Gamma}_{\text {per }}$ as $t$ goes to infinity.

Our proof of Theorem 1.1 relies on several linear and nonlinear estimates (product rules in Besov and Sobolev spaces) and uses Fourier series expansion of the time-periodic solution. Such an expansion was used in [13] for the time-periodic forced Navier-Stokes. The proof of Theorem 1.3 then goes through a fixed point argument in a suitable space. In order to have the asymptotic convergence, the functional space has to include decay properties. Thus, we are required to exhibit the decay from the velocity and the electro-magnetic fields. Both the dissipation coming from the viscosity of the fluid and from the resistivity in Ohm's law, enable us to have some decay for the velocity $\vec{u}$ and the electric field $\vec{E}$. To qualitatively transfer such a decay to the magnetic field is not as easy and clear as for $\vec{u}$ and $\vec{E}$. In [15], and then [11, a weak decay of the magnetic field was proven in both space dimension two and three. However, the decay was not used (in three space dimension) to construct global small solution. In the contrary, here the use of the decay is an essential fact to show asymptotic convergence.

\section{Construction of time-periodic solutions}

The purpose of this section is to prove Theorem 1.2 and Theorem 1.1. Since the method is the same for both but details are much more involved in critical spaces (the Besov case), and for the sake of simplicity, we opted to give the full details of the proof of Theorem 1.1 and only sketch the necessary changes to complete the proof of Theorem 1.2. First, we introduce some useful notation

\section{Notation :}

For $0<\delta<1$, we shall write

- $(\vec{u}, \vec{E}, \vec{B}) \in \mathbb{X}$ if

1. $\vec{u}$ belongs to $L_{\mathrm{per}}^{\infty} \dot{B}_{2, \infty}^{\frac{1}{2}} \cap L_{\mathrm{per}}^{2} \dot{H}^{\frac{3}{2}+\delta}$

2. $\vec{E}$ and $\vec{B}$ belong to $L_{\text {per }}^{\infty} H^{\frac{1}{2}+\delta}$.

- $(\vec{F}, \vec{G}, \vec{H}) \in \mathbb{Y}$ if

1. $\vec{F}$ belongs to $L_{\mathrm{per}}^{2} \dot{H}^{-\frac{1}{2}} \cap L_{\mathrm{per}}^{2} \dot{H}^{-\frac{1}{2}+\delta}$ 
2. the mean value $\vec{F}_{0}=\frac{1}{T} \int_{0}^{T} \vec{F}(t,) d$.$t belongs to \dot{B}_{2, \infty}^{-\frac{3}{2}}$

3. $\vec{G}$ belongs to $L_{\text {per }}^{2} H^{\frac{1}{2}+\delta}$

4. the mean value $\vec{G}_{0}=\frac{1}{T} \int_{0}^{T} \vec{G}(t,) d$.$t belongs to \dot{H}^{-1}$

5. $\vec{H}$ is divergence-free $(\operatorname{div} \vec{H}=0)$ and $\vec{H}$ belongs to $L_{\text {per }}^{2} H^{\frac{1}{2}+\delta}$

6. the mean value $\vec{H}_{0}=\frac{1}{T} \int_{0}^{T} \vec{H}(t,) d$.$t belongs to \dot{H}^{-2}$.

\subsection{Proof of theorem 1.1.}

The problem is solved by a Picard iterative scheme: $\left(\vec{u}_{\mathrm{per}}, \vec{E}_{\mathrm{per}}, \vec{B}_{\mathrm{per}}\right)$ will be the limit of the time-periodic functions $\left(\vec{U}_{n}, \vec{E}_{n}, \vec{B}_{n}\right)$ solving the system

$$
\left\{\begin{aligned}
\partial_{t} \vec{U}_{n+1}-\Delta \vec{U}_{n+1} & =\vec{F}_{n} \\
\partial_{t} \vec{E}_{n+1}-\vec{\nabla} \wedge \vec{B}_{n+1}+\vec{E}_{n+1} & =\vec{G}_{n} \\
\partial_{t} \vec{B}_{n+1}+\vec{\nabla} \wedge \vec{E}_{n+1} & =\vec{H}_{n} \\
\operatorname{div} \vec{u}_{n+1}=\operatorname{div} \vec{B}_{n+1} & =0
\end{aligned}\right.
$$

with

$$
\left\{\begin{aligned}
& \vec{U}_{0}=0, \quad \vec{E}_{0}=0, \vec{B}_{0}=0 \\
& \vec{F}_{n}=\mathbb{P}\left(-\operatorname{div}\left(\vec{U}_{n} \otimes \vec{U}_{n}\right)+\vec{E}_{n} \wedge \vec{B}_{n}+\left(\vec{U}_{n} \wedge \vec{B}_{n}\right) \wedge \vec{B}_{n}+\vec{F}_{\text {per }}\right) \\
& \vec{G}_{n}=-\vec{U}_{n} \wedge \vec{B}_{n}+\vec{G}_{\text {per }} \\
& \vec{H}_{n}=\vec{H}_{\text {per }}
\end{aligned}\right.
$$

where $\mathbb{P}$ is the Leray projection operator on solenoidal vector fields.

The first Lemma gives product rules in Sobolev spaces that close the iterative scheme. More precisely, we have.

Lemma 2.1. If $(\vec{u}, \vec{E}, \vec{B}) \in \mathbb{X}$ and $(\vec{F}, \vec{G}, \vec{H}) \in \mathbb{Y}$, define $\left(\vec{F}_{1}, \vec{G}_{1}, \vec{H}_{1}\right)$ as

$$
\left\{\begin{array}{l}
\vec{F}_{1}=\mathbb{P}(-\operatorname{div}(\vec{u} \otimes \vec{u})+\vec{E} \wedge \vec{B}+(\vec{u} \wedge \vec{B}) \wedge \vec{B}+\vec{F}) \\
\vec{G}_{1}=-\vec{u} \wedge \vec{B}+\vec{G} \\
\vec{H}_{1}=\vec{H}
\end{array}\right.
$$

Then we have $\left(\vec{F}_{1}, \vec{G}_{1}, \vec{H}_{1}\right) \in \mathbb{Y}$.

Proof. Point-wise product maps $\dot{B}_{2,1}^{3 / 2} \times H^{\frac{1}{2}+\delta}$ to $H^{\frac{1}{2}+\delta}$. As $L_{\mathrm{per}}^{\infty} \dot{B}_{2, \infty}^{\frac{1}{2}} \cap$ $L_{\text {per }}^{2} \dot{H}^{\frac{3}{2}+\delta} \subset L_{\text {per }}^{2} \dot{B}_{2,1}^{3 / 2}$, we find that $\vec{G}_{1}$ belongs to $L_{\text {per }}^{2} H^{\frac{1}{2}+\delta}$.

Moreover, pointwise product maps $\dot{B}_{2, \infty}^{1 / 2} \times L^{2}$ to $\dot{H}^{-1}$. Thus, we find that $\vec{u} \wedge \vec{B}$ belongs to $L_{\text {per }}^{\infty} \dot{H}^{-1}$ and the mean value of $\vec{G}_{1}$ belongs to $\dot{H}^{-1}$. 
For $s>0$, we have

$$
\|f g\|_{\dot{H}^{s}} \leq C_{s}\left(\|f\|_{\dot{B}_{2, \infty}^{1 / 2}}\|g\|_{\dot{H}^{s+1}}+\|g\|_{\dot{B}_{2, \infty}^{1 / 2}}\|f\|_{\dot{H}^{s+1}} .\right.
$$

Using the embedding $L_{\mathrm{per}}^{\infty} \dot{B}_{2, \infty}^{\frac{1}{2}} \cap L_{\mathrm{per}}^{2} \dot{H}^{\frac{3}{2}+\delta} \subset L_{\mathrm{per}}^{2} \dot{H}^{3 / 2}$, we find that the term $\operatorname{div}(\vec{u} \otimes \vec{u})$ belongs to $L_{\text {per }}^{2} \dot{H}^{-\frac{1}{2}} \cap L_{\text {per }}^{2} \dot{H}^{-\frac{1}{2}+\delta}$. Moreover, pointwise product maps $\dot{B}_{2, \infty}^{1 / 2} \times \dot{B}_{2, \infty}^{1 / 2}$ to $\dot{B}_{2, \infty}^{-1 / 2}$, so that div $(\vec{u} \otimes \vec{u})$ belongs to $L_{\text {per }}^{\infty} \dot{B}_{2, \infty}^{-3 / 2}$ and the mean value of $\operatorname{div}(\vec{u} \otimes \vec{u})$ belongs to $\dot{B}_{2, \infty}^{-3 / 2}$.

We have $\vec{E}+\vec{u} \wedge \vec{B} \in L_{\mathrm{per}}^{2} H^{\frac{1}{2}+\delta} \subset L_{\mathrm{per}}^{2} \dot{H}^{\frac{1}{2}} \cap L_{\mathrm{per}}^{2} \dot{H}^{\frac{1}{2}+\delta}$ and $B \in$ $L_{\text {per }}^{\infty} H^{\frac{1}{2}+\delta} \subset L_{\text {per }}^{\infty} \dot{H}^{1 / 2}$. This gives $(\vec{E}+\vec{u} \wedge \vec{B}) \wedge \vec{B} \in L_{\text {per }}^{2} \dot{H}^{-\frac{1}{2}} \cap L_{\text {per }}^{2} \dot{H}^{-\frac{1}{2}+\delta}$. Moreover, we have $\vec{E}+\vec{u} \wedge \vec{B} \in L_{\text {per }}^{2} H^{\frac{1}{2}+\delta} \subset L_{\text {per }}^{2} L^{2}$ and $B \in L_{\text {per }}^{\infty} H^{\frac{1}{2}+\delta} \subset$ $L_{\text {per }}^{\infty} L^{2}$, so that $(\vec{E}+\vec{u} \wedge \vec{B}) \wedge \vec{B} \in L_{\text {per }}^{2} L^{1} \subset L_{\text {per }}^{2} \dot{B}_{2, \infty}^{-3 / 2}$ and we find that the mean value of $(\vec{E}+\vec{u} \wedge \vec{B}) \wedge \vec{B}$ belongs to $\dot{B}_{2, \infty}^{-3 / 2}$. Thus, the lemma is proved.

The second Lemma shows that given a time-periodic forcing, we can construct, in the right functional space, a solution to the linear problem in the iterative scheme. Indeed,

\section{Lemma 2.2.}

Let $(\vec{F}, \vec{G}, \vec{H}) \in \mathbb{Y}$. Then the time-periodic solution $\vec{\Gamma}:=(\vec{u}, \vec{E}, \vec{B})$ of the system

$$
\left\{\begin{aligned}
\partial_{t} \vec{u}-\Delta \vec{u} & =\vec{F} \\
\partial_{t} \vec{E}-\vec{\nabla} \wedge \vec{B}+\vec{E} & =\vec{G} \\
\partial_{t} \vec{B}+\vec{\nabla} \wedge \vec{E} & =\vec{H} \\
\operatorname{div} \vec{B} & =0
\end{aligned}\right.
$$

satisfies $(\vec{u}, \vec{E}, \vec{B}) \in \mathbb{X}$.

Proof. We follow the formalism of Kyed [13] and expand $\vec{F}, \vec{G}, \vec{H}, \vec{u}, \vec{E}$ and 
$\vec{B}$ as time Fourier series:

$$
\left\{\begin{aligned}
\vec{F} & =\sum_{k \in \mathbb{Z}} \vec{F}_{k}(x) e^{i k \frac{2 \pi}{T} t} \\
\vec{G} & =\sum_{k \in \mathbb{Z}} \vec{G}_{k}(x) e^{i k \frac{2 \pi}{T} t} \\
\vec{H} & =\sum_{k \in \mathbb{Z}} \vec{H}_{k}(x) e^{i k \frac{2 \pi}{T} t} \\
\vec{u} & =\sum_{k \in \mathbb{Z}} \vec{U}_{k}(x) e^{i k \frac{2 \pi}{T} t} \\
\vec{E} & =\sum_{k \in \mathbb{Z}} \vec{E}_{k}(x) e^{i k \frac{2 \pi}{T} t} \\
\vec{B} & =\sum_{k \in \mathbb{Z}} \vec{B}_{k}(x) e^{i k \frac{2 \pi}{T} t} .
\end{aligned}\right.
$$

Define $\hat{F}_{k}, \hat{G}_{k}, \hat{H}_{k}, \hat{U}_{k}, \hat{E}_{k}, \hat{B}_{k}$ the Fourier transforms, in space, of $\vec{F}_{k}, \vec{G}_{k}, \vec{H}_{k}, \vec{U}_{k}, \vec{E}_{k}, \vec{B}_{k}$. First, we explicitly solve for $(\vec{U}, \vec{E}, \vec{B})$. From $\partial_{t} \vec{u}-\Delta \vec{u}=\vec{F}$, we get :

$$
\hat{U}_{k}(\xi)=\frac{1}{|\xi|^{2}+i \frac{2 \pi}{T} k} \hat{F}_{k}(\xi)
$$

From

$$
\partial_{t}^{2} \vec{B}=\partial_{t} \vec{H}-\vec{\nabla} \wedge \partial_{t} \vec{E}=\Delta \vec{B}-\vec{\nabla} \wedge \vec{G}+\vec{H}-\partial_{t} \vec{B}
$$

we get

$$
\hat{B}_{k}(\xi)=\frac{1}{|\xi|^{2}-\frac{4 \pi^{2}}{T^{2}} k^{2}+i \frac{2 \pi}{T} k}\left(\left(1+i \frac{2 \pi}{T} k\right) \hat{H}_{k}(\xi)-i \vec{\xi} \wedge \hat{G}_{k}(\xi)\right) .
$$

From $\partial_{t} \vec{E}+\vec{E}=\vec{G}+\vec{\nabla} \wedge \vec{B}$, we get

$$
\hat{E}_{k}(\xi)=\frac{1}{1+i \frac{2 \pi}{T} k}\left(\hat{G}_{k}(\xi)+i \vec{\xi} \wedge \hat{B}_{k}(\xi)\right) .
$$

If we decompose $\vec{E}_{k}$ into its solenoidal part $\vec{E}_{k, \sigma}$ and its irrotational part $\vec{E}_{k, \nabla}=\vec{\nabla} \frac{1}{\Delta} \operatorname{div} \vec{E}_{k}$, and similarly write $\vec{G}_{k}=\vec{G}_{k, \sigma}+\vec{G}_{k, \nabla}$, we get

$$
\hat{E}_{k, \sigma}(\xi)=\frac{1}{|\xi|^{2}-\frac{4 \pi^{2}}{T^{2}} k^{2}+i \frac{2 \pi}{T} k}\left(i \vec{\xi} \wedge \hat{H}_{k}(\xi)+i \frac{2 \pi}{T} k \hat{G}_{k, \sigma}(\xi)\right)
$$

and

$$
\hat{E}_{k, \nabla}(\xi)=\frac{1}{1+i \frac{2 \pi}{T} k} \hat{G}_{k, \nabla}(\xi) .
$$

Then, we proceed to estimate the solution. We are going to separately estimate the time averages $\vec{U}_{0}, \vec{E}_{0}$ and $\vec{B}_{0}$ and the fluctuation components 
$\vec{U}_{f}=\vec{u}-\vec{U}_{0}, \vec{E}_{f}=\vec{E}-\vec{E}_{0}$ and $\vec{B}_{f}=\vec{B}-\vec{B}_{0}$.

Notice that $\vec{U}_{0}=-\frac{1}{\Delta} \vec{F}_{0}$, and since $\vec{F}_{0} \in \dot{B}_{2, \infty}^{-3 / 2} \cap \dot{H}^{-\frac{1}{2}+\delta}$, we get $\vec{U}_{0} \in$ $\dot{B}_{2, \infty}^{1 / 2} \cap \dot{H}^{\frac{3}{2}+\delta}$.

Similarly, we have $\vec{B}_{0}=\frac{1}{\Delta} \vec{\nabla} \wedge \vec{G}_{0}-\frac{1}{\Delta} \vec{H}_{0}$. Since $\vec{G}_{0} \in \dot{H}^{-1} \cap H^{\frac{1}{2}+\delta}$ and $\vec{H}_{0} \in \dot{H}^{-2} \cap H^{\frac{1}{2}+\delta}$, we find that $\vec{B}_{0} \in H^{\frac{1}{2}+\delta}$.

We have $\vec{E}_{0}=\vec{G}_{0, \nabla}-\frac{1}{\Delta} \vec{\nabla} \wedge \vec{H}_{0}$. Since $\vec{G}_{0} \in H^{\frac{1}{2}+\delta}$ and $\vec{H}_{0} \in \dot{H}^{-2} \cap H^{\frac{1}{2}+\delta}$, we find that $\vec{E}_{0} \in H^{\frac{1}{2}+\delta}$.

Next, we estimate $\vec{U}_{f}$. We have

$$
\begin{aligned}
\int_{0}^{T}\left\|\vec{U}_{f}\right\|_{\dot{H}^{\frac{3}{2}+\delta}}^{2} d t & =T \sum_{k \neq 0}\left\|\vec{U}_{k}\right\|_{\dot{H}^{\frac{3}{2}+\delta}}^{2} \\
& =\frac{T}{(2 \pi)^{3}} \sum_{k \neq 0} \int_{\mathbb{R}^{3}} \frac{|\xi|^{3+2 \delta}}{|\xi|^{4}+\frac{4 \pi^{2}}{T^{2}} k^{2}}\left|\hat{F}_{k}(\xi)\right|^{2} d \xi \\
& =\frac{T}{(2 \pi)^{3}} \sum_{k \neq 0} \int_{\mathbb{R}^{3}} \frac{|\xi|^{4}}{|\xi|^{4}+\frac{4 \pi^{2}}{T^{2}} k^{2}}|\xi|^{-1+2 \delta}\left|\hat{F}_{k}(\xi)\right|^{2} d \xi \\
& \leq \int_{0}^{T}\left\|\vec{F}_{f}\right\|_{\dot{H}^{-\frac{1}{2}+\delta}}^{2} d t
\end{aligned}
$$

so that $\vec{U}_{f} \in L_{\mathrm{per}}^{2} \dot{H}^{\frac{3}{2}+\delta}$. On the other hand, we have :

$$
\begin{aligned}
\left\|\vec{U}_{f}(t, .)\right\|_{\dot{H}^{\frac{1}{2}}}^{2} & =\frac{1}{(2 \pi)^{3}} \int_{\mathbb{R}^{3}}|\xi|\left|\sum_{k \neq 0} \hat{U}_{k}(\xi) e^{i \frac{2 \pi}{T} k t}\right|^{2} d \xi \\
& \leq \frac{1}{(2 \pi)^{3}} \int_{\mathbb{R}^{3}}\left(\sum_{k \neq 0} \frac{|\xi|^{2}}{|\xi|^{4}+\frac{4 \pi^{2}}{T^{2}} k^{2}}\right)\left(\sum_{k \neq 0} \frac{\left|\hat{F}_{k}(\xi)\right|^{2}}{|\xi|}\right) d \xi \\
& \leq A_{T} \int_{0}^{T}\left\|\vec{F}_{f}\right\|_{\dot{H}^{-\frac{1}{2}}}^{2} d t
\end{aligned}
$$

with

$$
A_{T}=\frac{1}{T} \sup _{\xi \in \mathbb{R}^{3}} \sum_{k \neq 0} \frac{|\xi|^{2}}{|\xi|^{4}+\frac{4 \pi^{2}}{T^{2}} k^{2}} .
$$


Similarly, we have

$$
\begin{aligned}
\left\|\vec{B}_{f}(t, .)\right\|_{H^{\frac{1}{2}+\delta}}^{2} & =\frac{1}{(2 \pi)^{3}} \int_{\mathbb{R}^{3}}\left(1+|\xi|^{2}\right)^{\frac{1}{2}+\delta}\left|\sum_{k \neq 0} \hat{B}_{k}(\xi) e^{i \frac{2 \pi}{T} k t}\right|^{2} d \xi \\
\leq & \frac{2}{(2 \pi)^{3}} \int_{\mathbb{R}^{3}}\left(1+|\xi|^{2}\right)^{\frac{1}{2}+\delta}\left(\sum_{k \neq 0} \frac{|\xi|^{2}}{\left(|\xi|^{2}-\frac{4 \pi^{2}}{T^{2}} k^{2}\right)^{2}+\frac{4 \pi^{2}}{T^{2}} k^{2}}\right)\left(\sum_{k \neq 0}\left|\hat{G}_{k}(\xi)\right|^{2}\right) d \xi \\
& +\frac{2}{(2 \pi)^{3}} \int_{\mathbb{R}^{3}}\left(1+|\xi|^{2}\right)^{\frac{1}{2}+\delta}\left(\sum_{k \neq 0} \frac{1+\frac{4 \pi^{2}}{T^{2}} k^{2}}{\left(|\xi|^{2}-\frac{4 \pi^{2}}{T^{2}} k^{2}\right)^{2}+\frac{4 \pi^{2}}{T^{2}} k^{2}}\right)\left(\sum_{k \neq 0}\left|\hat{H}_{k}(\xi)\right|^{2}\right) d \xi \\
& \leq B_{T}\left(\int_{0}^{T}\left\|\vec{G}_{f}\right\|_{H^{\frac{1}{2}+\delta}}^{2} d t+\int_{0}^{T}\left\|\vec{H}_{f}\right\|_{H^{\frac{1}{2}+\delta}}^{2} d t\right)
\end{aligned}
$$

with

$$
B_{T}=\sup _{\xi \in \mathbb{R}^{3}} \frac{1}{T} \sum_{k \neq 0} \frac{1+|\xi|^{2}+\frac{4 \pi^{2}}{T^{2}} k^{2}}{\left(|\xi|^{2}-\frac{4 \pi^{2}}{T^{2}} k^{2}\right)^{2}+\frac{4 \pi^{2}}{T^{2}} k^{2}} .
$$

We have as well

$$
\begin{aligned}
\left\|\vec{E}_{\sigma, f}(t, .)\right\|_{H^{\frac{1}{2}+\delta}}^{2} & =\frac{1}{(2 \pi)^{3}} \int_{\mathbb{R}^{3}}\left(1+|\xi|^{2}\right)^{\frac{1}{2}+\delta}\left|\sum_{k \neq 0} \hat{E}_{\sigma, k}(\xi) e^{i \frac{2 \pi}{T} k t}\right|^{2} d \xi \\
\leq & \frac{2}{(2 \pi)^{3}} \int_{\mathbb{R}^{3}}\left(1+|\xi|^{2}\right)^{\frac{1}{2}+\delta}\left(\sum_{k \neq 0} \frac{|\xi|^{2}}{\left(|\xi|^{2}-\frac{4 \pi^{2}}{T^{2}} k^{2}\right)^{2}+\frac{4 \pi^{2}}{T^{2}} k^{2}}\right)\left(\sum_{k \neq 0}\left|\hat{H}_{k}(\xi)\right|^{2}\right) d \xi \\
& +\frac{2}{(2 \pi)^{3}} \int_{\mathbb{R}^{3}}\left(1+|\xi|^{2}\right)^{\frac{1}{2}+\delta}\left(\sum_{k \neq 0} \frac{\frac{4 \pi^{2}}{T^{2}} k^{2}}{\left(|\xi|^{2}-\frac{4 \pi^{2}}{T^{2}} k^{2}\right)^{2}+\frac{4 \pi^{2}}{T^{2}} k^{2}}\right)\left(\sum_{k \neq 0}\left|\hat{G}_{\sigma, k}(\xi)\right|^{2}\right) d \xi \\
\leq & C_{T}\left(\int_{0}^{T}\left\|\vec{G}_{\sigma, f}\right\|_{H^{\frac{1}{2}+\delta}}^{2} d t+\int_{0}^{T}\left\|\vec{H}_{f}\right\|_{H^{\frac{1}{2}+\delta}}^{2} d t\right)
\end{aligned}
$$

with

$$
C_{T}=\sup _{\xi \in \mathbb{R}^{3}} \frac{1}{T} \sum_{k \neq 0} \frac{|\xi|^{2}+\frac{4 \pi^{2}}{T^{2}} k^{2}}{\left(|\xi|^{2}-\frac{4 \pi^{2}}{T^{2}} k^{2}\right)^{2}+\frac{4 \pi^{2}}{T^{2}} k^{2}} .
$$

Finally, we have

$$
\begin{aligned}
\left\|\vec{E}_{\nabla, f}(t, .)\right\|_{H^{\frac{1}{2}+\delta}}^{2} & =\frac{1}{(2 \pi)^{3}} \int_{\mathbb{R}^{3}}\left(1+|\xi|^{2}\right)^{\frac{1}{2}+\delta}\left|\sum_{k \neq 0} \hat{E}_{\nabla, k}(\xi) e^{i \frac{2 \pi}{T} k t}\right|^{2} d \xi \\
& \leq \frac{1}{(2 \pi)^{3}} \int_{\mathbb{R}^{3}}\left(1+|\xi|^{2}\right)^{\frac{1}{2}+\delta}\left(\sum_{k \neq 0} \frac{1}{1+\frac{4 \pi^{2}}{T^{2}} k^{2}}\right)\left(\sum_{k \neq 0}\left|\hat{G}_{\sigma, k}(\xi)\right|^{2}\right) d \xi \\
& \leq D_{T} \int_{0}^{T}\left\|\vec{G}_{\nabla, f}\right\|_{H^{\frac{1}{2}+\delta}}^{2} d t
\end{aligned}
$$


with

$$
D_{T}=\sup _{\xi \in \mathbb{R}^{3}} \frac{1}{T} \sum_{k \neq 0} \frac{1}{1+\frac{4 \pi^{2}}{T^{2}} k^{2}} .
$$

Thus, in order to finish the proof of the lemma, we need only to check that $A_{T}, B_{T}, C_{T}$ and $D_{T}$ are finite. Equivalently, we must check that

$$
\alpha_{0}=\sup _{t \geq 0} \sum_{k=1}^{+\infty} \frac{t}{t^{2}+k^{2}}<+\infty
$$

and

$$
\beta_{0}=\sup _{t \geq 0} \sum_{k=1}^{+\infty} \frac{t+k^{2}}{k^{2}+\left(t-k^{2}\right)^{2}}<+\infty .
$$

If $t \leq 1 / 2$, we have $\frac{t}{t^{2}+k^{2}} \leq \frac{1}{2 k^{2}}$ and $\frac{t+k^{2}}{k^{2}+\left(t-k^{2}\right)^{2}} \leq \frac{3}{2 k^{2}}$ and the control of the sum is easy. Thus, we consider only the case $t>1 / 2$.

We write

$$
\sum_{k=1}^{+\infty} \frac{t}{t^{2}+k^{2}} \leq \sum_{1 \leq k \leq 2 t} \frac{t}{t^{2}}+\sum_{k>2 t} \frac{t}{k^{2}} \leq 2+t \min \left(\frac{1}{2 t-1}, 1+\frac{1}{2 t}\right) \leq \frac{7}{2} .
$$

Thus, $\alpha_{0}<+\infty$.

The case of $\beta_{0}$ is more delicate. We call $\Lambda(t)$ the set of integers $k$ such that $\left|t-k^{2}\right| \leq \frac{1}{4}\left(t+k^{2}\right)$. We have

$$
\sum_{k \notin \Lambda(t)} \frac{t+k^{2}}{k^{2}+\left(t-k^{2}\right)^{2}} \leq 16 \sum_{k=1}^{+\infty} \frac{t+k^{2}}{t^{2}+k^{4}} \leq 16\left(\frac{7}{2}+\frac{\pi^{2}}{6}\right) .
$$

We then must estimate $\sum_{k \in \Lambda(t)} \frac{t+k^{2}}{k^{2}+\left(t-k^{2}\right)^{2}}$. On $\Lambda(t)$, we have $k^{2} \in\left(\frac{3}{5} t, \frac{5}{3} t\right)$, thus $|k-\sqrt{t}|<\frac{1}{3} \sqrt{t}$ and $\left|k^{2}-t\right| \geq|k-\sqrt{t}| \frac{5}{3} \sqrt{t}$. This gives

$$
\sum_{k \in \Lambda(t)} \frac{t+k^{2}}{k^{2}+\left(t-k^{2}\right)^{2}} \leq \frac{8}{3} \sum_{k=1}^{\infty} \min \left(\frac{5}{3}, \frac{9}{25(k-\sqrt{t})^{2}}\right) \leq 5+\frac{8 \pi^{2}}{25} .
$$

\subsection{Another proof of Lemma 2.2: Energy-type estimate}

We give another proof of Lemma 2.2 :

Proof. We have, for $k \neq 0,\left.|| \xi\right|^{2}-\frac{4 \pi^{2}}{T^{2}} k^{2}+i \frac{2 \pi}{T} k\left|\geq \frac{2 \pi}{T}\right| k \mid$, and for $|\xi|>\frac{4 \pi}{T}|k|$, $\left.|| \xi\right|^{2}-\frac{4 \pi^{2}}{T^{2}} k^{2}+\left.i \frac{2 \pi}{T} k\left|\geq \frac{3}{4}\right| \xi\right|^{2}$. Thus, it is straightforward that the solution $(\vec{u}, \vec{E}, \vec{B})$ of system (2.4) satisfies

$$
\int_{0}^{T}\left\|\vec{E}_{f}\right\|_{H^{1 / 2+\delta}}^{2}+\left\|\vec{B}_{f}\right\|_{H^{1 / 2+\delta}}^{2} d t \leq C \int_{0}^{T}\left\|\vec{G}_{f}\right\|_{H^{1 / 2+\delta}}^{2}+\left\|\vec{H}_{f}\right\|_{H^{1 / 2+\delta}}^{2} d t .
$$


Now, if we assume that $\vec{G}_{f}$ and $\vec{H}_{f}$ are trigonometric polynomials with respect to the time variable and with values, in $H^{3 / 2+\delta}$, we find that $\vec{E}_{f}$ and $\vec{B}_{f}$ are in $\mathcal{C}_{\text {per }} H^{1 / 2+\delta}$ and that $\partial_{t} \vec{E}_{f}$ and $\partial_{t} \vec{f}$ belong to $L_{\text {per }}^{2} H^{\frac{1}{2}+\delta}$.. Moreover, we have, writing $\overrightarrow{\mathcal{E}}=(I d-\Delta)^{\frac{1}{4}+\frac{\delta}{2}} \vec{E}_{f}$ and $\overrightarrow{\mathcal{B}}=(I d-\Delta)^{\frac{1}{4}+\frac{\delta}{2}} \vec{B}_{f}$,

$$
\begin{gathered}
\frac{d}{d t}\left(\frac{\left\|\vec{E}_{f}\right\|_{H^{\frac{1}{2}+\delta}}^{2}+\left\|\vec{B}_{f}\right\|_{H^{\frac{1}{2}+\delta}}^{2}}{2}\right)=\int_{\mathbb{R}^{3}} \partial_{t} \overrightarrow{\mathcal{E}} \cdot \overrightarrow{\mathcal{E}}+\partial_{t} \overrightarrow{\mathcal{B}} \cdot \overrightarrow{\mathcal{B}} d x \\
=\int_{\mathbb{R}^{3}} \overrightarrow{\mathcal{E}} \cdot\left((I d-\Delta)^{\frac{1}{4}+\frac{\delta}{2}} G_{f}-\overrightarrow{\mathcal{E}}+\vec{\nabla} \wedge \overrightarrow{\mathcal{B}}\right)+\overrightarrow{\mathcal{B}} \cdot\left((I d-\Delta)^{\frac{1}{4}+\frac{\delta}{2}} \vec{H}_{f}-\vec{\nabla} \wedge \overrightarrow{\mathcal{E}}\right) d x \\
=\int_{\mathbb{R}^{3}} \overrightarrow{\mathcal{E}} \cdot\left((I d-\Delta)^{\frac{1}{4}+\frac{\delta}{2}} G_{f}-\overrightarrow{\mathcal{E}}\right)+\overrightarrow{\mathcal{B}} \cdot(I d-\Delta)^{\frac{1}{4}+\frac{\delta}{2}} \vec{H}_{f} d x \\
\leq\left\|\vec{E}_{f}\right\|_{H^{1 / 2}+\delta}\left\|\vec{G}_{f}\right\|_{H^{1+2+\delta}}+\left\|\vec{B}_{f}\right\|_{H^{1 / 2+\delta}}\left\|\vec{H}_{f}\right\|_{H^{1+2+\delta}}
\end{gathered}
$$

This gives, for $-T \leq t_{0} \leq 0 \leq t \leq T$

$$
\begin{aligned}
\left\|\vec{E}_{f}(t, .)\right\|_{H^{\frac{1}{2}+\delta}}^{2}+\left\|\vec{B}_{f}(t, .)\right\|_{H^{\frac{1}{2}+\delta}}^{2} & \leq\left\|\vec{E}_{f}\left(t_{0}, . .\right)\right\|_{H^{\frac{1}{2}+\delta}}^{2}+\left\|\vec{B}_{f}\left(t_{0}, .\right)\right\|_{H^{\frac{1}{2}+\delta}}^{2} \\
& +2 \int_{-T}^{T}\left\|\vec{E}_{f}\right\|_{H^{1 / 2+\delta}}\left\|\vec{G}_{f}\right\|_{H^{1+2+\delta}} d s \\
& +2 \int_{-T}^{T}\left\|\vec{B}_{f}\right\|_{H^{1 / 2+\delta}}\left\|\vec{H}_{f}\right\|_{H^{1+2+\delta}} d s
\end{aligned}
$$

Integrating with respect to $t_{0}$, we find

$$
\begin{aligned}
\left\|\vec{E}_{f}(t, .)\right\|_{H^{\frac{1}{2}+\delta}}^{2}+\left\|\vec{B}_{f}(t, .)\right\|_{H^{\frac{1}{2}+\delta}}^{2} & \leq \frac{1}{T} \int_{0}^{T}\left\|\vec{E}_{f}\left(t_{0}, . .\right)\right\|_{H^{\frac{1}{2}+\delta}}^{2}+\left\|\vec{B}_{f}\left(t_{0}, .\right)\right\|_{H^{\frac{1}{2}+\delta}}^{2} d t_{0} \\
& +4 \int_{0}^{T}\left\|\vec{E}_{f}\right\|_{H^{1 / 2+\delta}}\left\|\vec{G}_{f}\right\|_{H^{1+2+\delta}} d s \\
& +4 \int_{0}^{T}\left\|\vec{B}_{f}\right\|_{H^{1 / 2+\delta}}\left\|\vec{H}_{f}\right\|_{H^{1+2+\delta}} d s
\end{aligned}
$$

and finally

$$
\left.\left\|\vec{E}_{f}(t, .)\right\|_{H^{\frac{1}{2}+\delta}}^{2}+\left\|\vec{B}_{f}(t, .)\right\|_{H^{\frac{1}{2}+\delta}}^{2} \leq C \int_{0}^{T}\left\|\vec{G}_{f}\right\|_{H^{1+2+\delta}}^{2}+\left\|\vec{H}_{f}\right\|_{H^{1+2+\delta}}^{2} d s\right) .
$$

We then conclude the proof of the lemma by a density argument.

Remark 2.1. In the proof of Theorem 1.1, we actually show that $\vec{U}_{f} \in$ $\mathcal{C}_{\text {per }} \dot{H}^{1 / 2} \cap L_{\text {per }}^{2} \dot{H}^{3 / 2+\delta}$ and, $\vec{E} \in \mathcal{C}_{\text {per }} H^{1 / 2+\delta}$ and $\vec{B} \in \mathcal{C}_{\text {per }} H^{1 / 2+\delta}$ while $\vec{U}_{0} \in \dot{B}_{2, \infty}^{1 / 2} \cap \dot{H}^{3 / 2+\delta}$. Thus the most inconvenient term to deal with is thus the mean value $\vec{U}_{0}=\frac{1}{T} \int_{0}^{T} \vec{u}(t,) d$.$t .$ 


\subsection{Proof of Theorem 1.2}

In this part, we only sketch the proof Theorem 1.2 as the steps are basically similar to those of Theorem 1.1. First, we shall adjust the previous spaces and define

- $(\vec{u}, \vec{E}, \vec{B}) \in \tilde{\mathbb{X}}$ if

1. $\vec{u}$ belongs to $\tilde{L}_{\mathrm{per}}^{\infty} \dot{B}_{2,(\infty, 1)}^{\frac{1}{2}} \cap \tilde{L}_{\mathrm{per}}^{2} \dot{B}_{2,(\infty, 1)}^{\frac{3}{2}}$

2. $\vec{E}$ and $\vec{B}$ belong to $\tilde{L}_{\text {per }}^{\infty} H^{\frac{1}{2}}$.

- $(\vec{F}, \vec{G}, \vec{H}) \in \tilde{\mathbb{Y}}$ if

1. $\vec{F}$ belongs to $\tilde{L}_{\text {per }}^{2} \dot{B}_{2,(\infty, 1)}^{-\frac{1}{2}}$

2. the mean value $\vec{F}_{0}=\frac{1}{T} \int_{0}^{T} \vec{F}(t,) d$.$t belongs to \dot{B}_{2,(\infty, 1)}^{-\frac{3}{2}}$

3. $\vec{G}$ belongs to $L_{\mathrm{per}}^{2} H^{\frac{1}{2}}$

4. the mean value $\vec{G}_{0}=\frac{1}{T} \int_{0}^{T} \vec{G}(t,) d$.$t belongs to \dot{H}^{-1}$

5. $\vec{H}$ is divergence-free $(\operatorname{div} \vec{H}=0)$ and $\vec{H}$ belongs to $L_{\text {per }}^{2} H^{\frac{1}{2}}$

6. the mean value $\vec{H}_{0}=\frac{1}{T} \int_{0}^{T} \vec{H}(t,) d$.$t belongs to \dot{H}^{-2}$.

Lemma 2.1 can be extended to the following result in the case of critical Besov spaces. Again, it is sufficient to treat point-wise estimates (at fixed time).

\section{Lemma 2.3.}

$$
\begin{array}{r}
H^{\frac{1}{2}} \times H^{\frac{1}{2}} \hookrightarrow \dot{B}_{2,1}^{-\frac{1}{2}} \hookrightarrow \dot{B}_{2,(\infty, 1)}^{-\frac{1}{2}} \\
\left(\dot{B}_{2,(\infty, 1)}^{\frac{1}{2}} \cap \dot{B}_{2,(\infty, 1)}^{\frac{3}{2}}\right) \times H^{\frac{1}{2}} \hookrightarrow H^{\frac{1}{2}} \\
\left(\dot{B}_{2,(\infty, 1)}^{\frac{1}{2}} \cap \dot{B}_{2,(\infty, 1)}^{\frac{3}{2}}\right) \times\left(\dot{B}_{2,(\infty, 1)}^{\frac{1}{2}} \cap \dot{B}_{2,(\infty, 1)}^{\frac{3}{2}}\right) \hookrightarrow \dot{B}_{2,(\infty, 1)}^{\frac{1}{2}} .
\end{array}
$$

Proof. The first product is classical and we omit it here. To prove the other two, we first observe that

$$
\left(\dot{B}_{2,(\infty, 1)}^{\frac{1}{2}} \cap \dot{B}_{2,(\infty, 1)}^{\frac{3}{2}}\right) \hookrightarrow L^{\infty} .
$$

Let $u \in \dot{B}_{2,(\infty, 1)}^{\frac{1}{2}} \cap \dot{B}_{2,(\infty, 1)}^{\frac{3}{2}}$, and $B \in H^{\frac{1}{2}}$. We begin by estimating the term $T_{u} B$ in the para-product. We have

$$
\left\|\Delta_{q} T_{u} B\right\|_{L^{2}} \leq\|u\|_{L^{\infty}}\left\|\Delta_{q} B\right\|_{L^{2}} \lesssim\|u\|_{\dot{B}_{2,(\infty, 1)}^{\frac{1}{2}} \cap \dot{B}_{2,(\infty, 1)}^{\frac{3}{2}}}\left\|\Delta_{q} B\right\|_{L^{2}}
$$


giving

$$
\left\|T_{u} B\right\|_{H^{\frac{1}{2}}} \lesssim\|u\|_{\dot{B}_{2,(\infty, 1)}^{\frac{1}{2}} \cap \dot{B}_{2,(\infty, 1)}^{\frac{3}{2}}}\|B\|_{H^{\frac{1}{2}}} \cdot
$$

When $k \leq 0$, by Bernstein's lemma we have

$$
\begin{aligned}
2^{\frac{k}{2}} \sum_{j \leq k} 2^{\frac{3 j}{2}}\left\|\Delta_{j} B\right\|_{L^{2}}\left\|\Delta_{k} u\right\|_{L^{2}} & \leq \sum_{j \leq k} 2^{\frac{j}{2}}\left\|\Delta_{j} B\right\|_{L^{2}} 2^{j-k} 2^{\frac{3 k}{2}}\left\|\Delta_{k} u\right\|_{L^{2}} \\
& \lesssim\|u\|_{\dot{B}_{2,(\infty, 1)}^{\frac{1}{2}}} \sum_{j \leq k} 2^{\frac{j}{2}}\left\|\Delta_{j} B\right\|_{L^{2}} 2^{j-k}
\end{aligned}
$$

For $k \geq 1$, we decompose further as follows

$$
\sum_{j \leq k} 2^{\frac{3 j}{2}}\left\|\Delta_{j} B\right\|_{L^{2}}\left\|\Delta_{k} u\right\|_{L^{2}} \leq \sum_{j \leq 0}(\cdot)+\sum_{0 \leq j \leq k}(\cdot)
$$

and estimate the terms as below

$$
2^{\frac{k}{2}} \sum_{j \leq 0}(\cdot) \lesssim\|B\|_{H^{\frac{1}{2}}} 2^{\frac{k}{2}}\left\|\Delta_{k} u\right\|_{L^{2}}
$$

and

$$
2^{\frac{k}{2}} \sum_{0 \leq j \leq k}(\cdot) \lesssim 2^{\frac{3 k}{2}}\left\|\Delta_{k} u\right\|_{L^{2}} \sum_{0 \leq j \leq k} 2^{\frac{j}{2}}\left\|\Delta_{j} B\right\|_{L^{2}} 2^{j-k} .
$$

Using Young's inequality, we conclude that

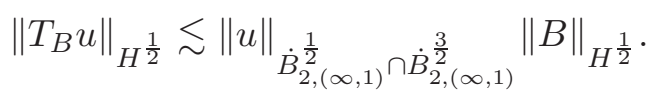

Finally we estimate the remainder term $R(u, B)$ only when $k \leq 0$ because the case $k \geq 1$ is easier. By Bernstein's lemma we have

$$
\begin{aligned}
2^{\frac{k}{2}} \sum_{j \geq k-2} 2^{\frac{3 q}{2}}\left\|\Delta_{j} B\right\|_{L^{2}}\left\|\Delta_{j} u\right\|_{L^{2}} & \leq 2^{2 k} \sum_{j \leq 0}\left\|\Delta_{j} u\right\|_{L^{2}}\left\|\Delta_{j} B\right\|_{L^{2}} \\
& +2^{2 k} \sum_{k-2 \leq j \leq 0}\left\|\Delta_{j} B\right\|_{L^{2}}\left\|\Delta_{j} u\right\|_{L^{2}} \\
& \lesssim\|u\|_{\dot{B}_{2,(\infty, 1)}^{\frac{1}{2}}\|B\|_{L^{2}} 2^{2 k}} \\
& +\sum_{j \geq k-2} 2^{\frac{3 j}{2}}\left\|\Delta_{j} u\right\|_{L^{2}} 2^{\frac{j}{2}}\left\|\Delta_{j} B\right\|_{L^{2}} 2^{2(k-j)}
\end{aligned}
$$

Thus we conclude that

$$
\|R(u, B)\|_{H^{\frac{1}{2}}} \lesssim\|u\|_{\dot{B}_{2,(\infty, 1)}^{\frac{1}{2}} \cap \dot{B}_{2,(\infty, 1)}^{\frac{3}{2}}}\|B\|_{H^{\frac{1}{2}}}
$$

as required. The proof of estimate (2.12) is similar. As before we have

$$
\left\|\Delta_{q} T_{u} u\right\|_{L^{2}} \leq\|u\|_{L^{\infty}}\left\|\Delta_{q} u\right\|_{L^{2}} \lesssim\|u\|_{\dot{B}_{2,(\infty, 1)}^{\frac{1}{2}} \cap \dot{B}_{2,(\infty, 1)}^{\frac{3}{2}}}\left\|\Delta_{q} u\right\|_{L^{2}}
$$


giving

$$
\left\|T_{u} u\right\|_{\dot{B}_{2,(\infty, 1)}^{\frac{1}{2}}} \lesssim\|u\|_{\dot{B}_{2,(\infty, 1)}^{\frac{1}{2}} \cap \dot{B}_{2,(\infty, 1)}^{\frac{3}{2}}}\|u\|_{\dot{B}_{2,(\infty, 1)}^{\frac{1}{2}}} .
$$

Next we estimate the remainder term only for $k \leq 0$, by Bernstein's lemma we have

$$
\begin{aligned}
2^{\frac{k}{2}} \sum_{j \geq k-2} 2^{\frac{3 q}{2}}\left\|\Delta_{j} u\right\|_{L^{2}}\left\|\Delta_{j} u\right\|_{L^{2}} & \leq 2^{2 k} \sum_{j \leq 0}\left\|\Delta_{j} u\right\|_{L^{2}}\left\|\Delta_{j} u\right\|_{L^{2}} \\
& +2^{2 k} \sum_{k-2 \leq j \leq 0}\left\|\Delta_{j} u\right\|_{L^{2}}\left\|\Delta_{j} u\right\|_{L^{2}} \\
& \lesssim\|u\|_{\dot{B}_{2,(\infty, 1)}^{2}}^{2} 2^{2 k} \\
& +\sum_{j \geq k-2} 2^{\frac{3 j}{2}}\left\|\Delta_{j} u\right\|_{L^{2}} 2^{\frac{j}{2}}\left\|\Delta_{j} u\right\|_{L^{2}} 2^{2(k-j)} .
\end{aligned}
$$

Consequently, we have

$$
\|R(u, u)\|_{H^{\frac{1}{2}}} \lesssim\|u\|_{\dot{B}_{2,(\infty, 1)}^{\frac{1}{2}}}^{2} \cap \dot{B}_{2,(\infty, 1)}^{\frac{3}{2}} .
$$

as desired.

Now, we give a result equivalent to Lemma 2.2

\section{Lemma 2.4.}

Let $(\vec{F}, \vec{G}, \vec{H}) \in \tilde{\mathbb{Y}}$. Then the time-periodic solution $\vec{\Gamma}:=(\vec{u}, \vec{E}, \vec{B})$ of the system

$$
\left\{\begin{aligned}
\partial_{t} \vec{u}-\Delta \vec{u} & =\vec{F} \\
\partial_{t} \vec{E}-\vec{\nabla} \wedge \vec{B}+\vec{E} & =\vec{G} \\
\partial_{t} \vec{B}+\vec{\nabla} \wedge \vec{E} & =\vec{H} \\
\operatorname{div} \vec{B} & =0
\end{aligned}\right.
$$

satisfies $(\vec{u}, \vec{E}, \vec{B}) \in \tilde{\mathbb{X}}$.

Proof. We only estimate the solution $\hat{U}_{k}(\xi)=\frac{1}{|\xi|^{2}+i \frac{2 \pi}{T} k} \hat{F}_{k}(\xi)$ in $\tilde{L}^{2}\left(\dot{B}_{2,(\infty, 1)}^{\frac{3}{2}}\right)$, as all the other estimates are similar. First, we have

$$
\left\|\Delta_{q} \vec{U}\right\|_{L^{2}\left(0, T ; L^{2}\right)} \lesssim \sqrt{\sum_{k \in \mathbb{Z} \backslash 0} \frac{\left\|\Delta_{q} \vec{F}_{k}\right\|_{L^{2}}^{2}}{2^{4 q}+\frac{k^{2}}{T^{2}}} .}
$$

Hence,

$$
\begin{aligned}
\sup _{q \leq 0} 2^{\frac{3 q}{2}}\left\|\Delta_{q} \vec{U}\right\|_{L^{2}\left(0, T ; L^{2}\right)} & \lesssim \sup _{q \leq 0} 2^{-\frac{q}{2}} \sqrt{\sum_{k \in \mathbb{Z}}\left\|\Delta_{q} \vec{F}_{k}\right\|_{L^{2}}^{2}} \sqrt{\sup _{q \leq 0} \sup _{k \in \mathbb{Z} \backslash 0} \frac{2^{4 q}}{2^{4 q}+\frac{k^{2}}{T^{2}}}} \\
& \lesssim \sup _{q \leq 0} 2^{-\frac{q}{2}} \sqrt{\sum_{k \in \mathbb{Z} \backslash 0}\left\|\Delta_{q} \vec{F}_{k}\right\|_{L^{2}}^{2}} \lesssim\|F\|_{\tilde{L}^{2}\left(\dot{B}_{2,(\infty, 1)}^{-\frac{1}{2}}\right)},
\end{aligned}
$$


and similarly

$$
\begin{aligned}
\sum_{q \geq 1} 2^{\frac{3 q}{2}}\left\|\Delta_{q} \vec{U}\right\|_{L^{2}\left((0, T) ; L^{2}\right)} & \lesssim \sum_{q \leq 0} 2^{-\frac{q}{2}} \sqrt{\sum_{k \in \mathbb{Z} \backslash 0}\left\|\Delta_{q} \vec{F}_{k}\right\|_{L^{2}}^{2}} \sqrt{\sup _{q \leq 0} \sup _{k \in \mathbb{Z} \backslash 0} \frac{2^{4 q}}{2^{4 q}+\frac{k^{2}}{T^{2}}}} \\
& \lesssim \sum_{q \geq 1} 2^{-\frac{q}{2}} \sqrt{\sum_{k \in \mathbb{Z} \backslash 0}\left\|\Delta_{q} \vec{F}_{k}\right\|_{L^{2}}^{2}} \lesssim\|F\|_{\tilde{L}^{2}\left(\dot{B}_{2,(\infty, 1)}^{-\frac{1}{2}}\right)}
\end{aligned}
$$

\section{Asymptotic stability}

The purpose of this section is to prove Theorem 1.3. In the sequel, we omit the $\vec{s}$ symbol in order to alleviate the notation, since we will use also ${ }^{\wedge}$ for the Fourier transform, $\sim$ etc.

Denote by $\Gamma_{\text {per }}$ the $T$-periodic small solution of (0.1) given by Theorem 1.2 , We decompose $\bar{\Gamma}$ the solution of (0.1) as

$$
\bar{\Gamma}:=\Gamma_{\text {per }}+\Gamma_{\text {err }}
$$

where the "error" term $\Gamma_{\text {err }}$ is further decomposed as $\Gamma_{\text {err }}=e^{t \mathcal{A}} \Gamma_{\text {err }}^{0}+\Gamma$. We assume that the initial data $\Gamma_{\text {err }}^{0}$ is small in the space $\dot{\mathcal{B}}_{2,(\infty, 1)}^{\frac{1}{2}} \times H^{\frac{1}{2}} \times H^{\frac{1}{2}}$, and $\Gamma^{0}=0$. It is easy to see that $\Gamma_{\text {err }}$ solves the following integral equation

$$
\Gamma_{\mathrm{err}}(t)=e^{t \mathcal{A}} \Gamma_{\mathrm{err}}^{0}+\int_{0}^{t} e^{\left(t-t^{\prime}\right) \mathcal{A}} \mathcal{N}\left(\Gamma_{\mathrm{err}}\left(t^{\prime}\right)\right) d t^{\prime}
$$

with

$$
\mathcal{A}=\left(\begin{array}{ccc}
\Delta & 0 & 0 \\
0 & -I & \nabla \wedge \\
0 & -\nabla \wedge \cdot & 0
\end{array}\right)
$$

and the three components of the nonlinearity $\mathcal{N}=\left(\mathcal{N}_{1}, \mathcal{N}_{2}, \mathcal{N}_{3}\right)$ are

$$
\begin{aligned}
& \mathcal{N}_{1}=\mathbb{P}(-\nabla(u \otimes u)-\nabla\left(u_{\text {per }} \otimes u\right)-\nabla\left(u \otimes u_{\text {per }}\right) \\
&+ E \times B+E \times B_{\text {per }}+E_{\text {per }} \times B \\
&+(u \times B) \times B+\left(u \times B_{\text {per }}\right) \times B_{\text {per }} \\
&+ {\left.\left[u_{\text {per }} \times B\right] \times B_{\text {per }}+\left[u_{\text {per }} \times B_{\text {per }}\right] \times B\right), } \\
& \\
& \mathcal{N}_{2}=u \times B+u \times B_{\text {per }}+u_{\text {per }} \times B
\end{aligned}
$$

and $\mathcal{N}_{3}=0$, respectively. Observe that the nonlinear term is expressed only in terms of the periodic solution. The construction of $\Gamma$ follows a standard fixed point argument. 
Let $B_{\delta}$ be the ball of the space $\mathcal{X}$ centred at zero and with radius $\delta>0$ to be chosen. On that ball, define the map $\Phi$ as follows

$$
\begin{aligned}
\Phi: B_{\delta} \subset \mathcal{X} & \longrightarrow \mathcal{X} \\
\Gamma & \mapsto \Phi(\Gamma):=\int_{0}^{t} e^{\left(t-t^{\prime}\right) \mathcal{A}} \mathcal{N}\left(e^{t^{\prime} \mathcal{A}} \Gamma_{\mathrm{err}}^{0}+\Gamma\left(t^{\prime}\right)\right) d t^{\prime} .
\end{aligned}
$$

Hence, the result of Theorem 1.3 will be a consequence of the following proposition.

Proposition 3.1. If $\left\|\Gamma_{\mathrm{err}}^{0}\right\|_{\mathcal{B}_{2,(\infty, 1)}^{\frac{1}{2}} \times H^{\frac{1}{2}} \times H^{\frac{1}{2}}} \leq \kappa \delta$, with $\delta>0$ and $\kappa>0$ sufficiently small, then the map $\Phi$ is a contraction on $B_{\delta}$.

Indeed, admitting for now this proposition, Picard's theorem gives the existence of a fixed point of the map $\Phi$, call it $\Gamma$. Then clearly $e^{t \mathcal{A}} \Gamma_{\mathrm{err}}^{0}+\Gamma(t)$ would be the desired solution of (0.1).

In order to prove Proposition 3.1, we need a few preliminary lemmas.

\subsection{Preliminary results}

We start with several preliminary lemmas. First, we prove the following result of parabolic regularity, in the spirit of [2], adapted to the spaces $\mathcal{X}$ and $\mathcal{Y}_{1}$ in the following way.

Lemma 3.1 (Adapted maximum parabolic regularity). Let $u$ be a smooth divergence free vector field solving

$$
\left\{\begin{array}{l}
\partial_{t} u-\Delta u+\nabla p=f \\
u_{\mid t=0}=u^{0}
\end{array}\right.
$$

on some time interval $[0, T]$. Then, we have

$$
\|u\|_{\mathcal{X}_{1} \cap \tilde{L}^{\infty}\left(\dot{B}_{2,(\infty, 1)}^{\frac{1}{2}}\right)} \lesssim\left\|u^{0}\right\|_{\dot{B}_{2,(\infty, 1)}^{\frac{1}{2}}}+\|f\|_{\mathcal{Y}_{1}} .
$$

Proof. From the start, for any $k \in \mathbb{Z}$, and $0 \leq \varepsilon \leq 1$ denote by

$$
\alpha_{k}:=\min \left(2^{k}, 1\right),
$$

and define the norm

$$
M_{k, \varepsilon}(f):=\sup _{n \in \mathbb{N}}\left(2^{-k\left(\frac{1}{2}+\varepsilon\right)}(n+1)^{\frac{1-\varepsilon}{2}}\left\|\Delta_{k} f\right\|_{L_{t}^{2}\left(n, n+1 ; L_{x}^{2}\right)}\right),
$$

to be used to control the low frequencies of $f$, and

$$
\tilde{M}_{k, \varepsilon}(f):=\sup _{n \in \mathbb{N}}\left(2^{-\frac{k}{2}}(n+1)^{\frac{1-\varepsilon}{2}}\left\|\Delta_{k} f\right\|_{L_{t}^{2}\left(n, n+1 ; L_{x}^{2}\right)}\right),
$$


to control its high frequencies. Also, observe that for all $c>0$ we have

$$
x^{1-\varepsilon} e^{-c x^{2}} \leq C(c, \varepsilon) \lesssim 1 .
$$

Moreover, the following elementary estimate

$$
\int_{0}^{A} e^{u^{2}} d u \leq \frac{e^{A}-1}{A}
$$

clearly implies the following one

$$
\sup _{t>0} t^{\frac{1}{2}} e^{-\frac{t}{2}} \int_{0}^{t} \frac{e^{\frac{s}{2}}}{\sqrt{s}} d s \leq 4
$$

which will be of frequent use in the proofs of our linear estimates. In addition, the following estimate is classical and can be found, for example, in [2]

$$
\left\|\Delta_{k} e^{t \Delta} u_{0}\right\|_{L^{2}} \lesssim e^{-c t 2^{2 k}}\left\|\Delta_{k} u_{0}\right\|_{L^{2}}
$$

From now on, we will "drop" this constant $c$ by taking it always equals to one.

Duhamel's formula for the solution of (3.2) gives

$$
u(t)=e^{t \Delta} u_{0}+\int_{0}^{t} e^{(t-s) \Delta} \mathbb{P} f(s) d s,
$$

where $\mathbb{P}$ is Leray's projection.

- First, we focus on the homogeneous solution $e^{t \Delta} u_{0}$. Multiplying (3.5) by $2^{\frac{k}{2}}$, taking the supremum in time and then summing in $k$ (and the supremum in $k$ for low frequencies), easily gives

$$
\operatorname{supp}_{t>0}\left\|e^{t \Delta} u_{0}\right\|_{\dot{\mathcal{B}}_{2,(\infty, 1)}^{\frac{1}{2}}} \lesssim\left\|u_{0}\right\|_{\dot{\mathcal{B}}_{2,(\infty, 1)}^{\frac{1}{2}}} .
$$

Now we focus on the norm giving the decay. From (3.5) and for all $0 \leq \varepsilon \leq 1$, we have for any $k \leq 0$

$$
\begin{aligned}
(t+1)^{\frac{1-\varepsilon}{2}} 2^{k\left(\frac{3}{2}-\varepsilon\right)}\left\|\Delta_{k} e^{t \Delta} u_{0}\right\|_{L^{2}} & \lesssim\left(2^{2 k}(t+1)\right)^{\frac{1-\varepsilon}{2}} e^{-c t 2^{2 k}} 2^{\frac{k}{2}}\left\|\Delta_{k} u_{0}\right\|_{L^{2}} \\
& \lesssim\left(2^{2 k}(t+1)\right)^{\frac{1-\varepsilon}{2}} e^{-(t+1) 2^{2 k}} 2^{\frac{k}{2}}\left\|\Delta_{k} u_{0}\right\|_{L^{2}} \\
& \lesssim 2^{\frac{k}{2}}\left\|\Delta_{k} u_{0}\right\|_{L^{2}}
\end{aligned}
$$

where we used (3.3). When $k \geq 1$, we estimate as follows 


$$
\begin{aligned}
(t+1)^{\frac{1-\varepsilon}{2}} 2^{\frac{k}{2}}\left\|\Delta_{k} e^{t \Delta} u_{0}\right\|_{L^{2}} & \lesssim(t+1)^{\frac{1-\varepsilon}{2}} e^{-t 2^{2 k}} 2^{\frac{k}{2}}\left\|\Delta_{k} u_{0}\right\|_{L^{2}} \\
& \lesssim(t+1)^{\frac{1-\varepsilon}{2}} e^{-(t+1)} 2^{\frac{k}{2}}\left\|\Delta_{k} u_{0}\right\|_{L^{2}} \\
& \lesssim 2^{\frac{k}{2}}\left\|\Delta_{k} u_{0}\right\|_{L^{2}}
\end{aligned}
$$

Obviously, (3.7) and (3.8) give

$$
\operatorname{supp}_{t>0}(t+1)^{\frac{1-\varepsilon}{2}}\left\|e^{t \Delta} u_{0}\right\|_{\dot{\mathcal{B}}_{2,(\infty, 1)}^{\frac{3}{2}-\varepsilon, \frac{1}{2}}} \lesssim\left\|u_{0}\right\|_{\dot{\mathcal{B}}_{2,(\infty, 1)}^{\frac{1}{2}}} .
$$

Again thanks to (3.5), we have

$$
\begin{aligned}
\left\|\Delta_{k} e^{t \Delta} u_{0}\right\|_{L^{2}\left(n, n+1, L_{x}^{2}\right)} & \lesssim \frac{\sqrt{e^{-2 n 2^{2 k}}-e^{-2(n+1) 2^{2 k}}}}{2^{k}}\left\|\Delta_{k} u_{0}\right\|_{L^{2}} \\
& \lesssim 2^{-k} \alpha_{k} e^{-n 2^{2 k}}\left\|\Delta_{k} u_{0}\right\|_{L^{2}},
\end{aligned}
$$

so that

$(n+1)^{\frac{1-\varepsilon}{2}} 2^{\frac{3}{2} k}\left\|\Delta_{k} u\right\|_{L^{2}\left(n, n+1, L_{x}^{2}\right)} \lesssim(n+1)^{\frac{1-\varepsilon}{2}} e^{-(n+1)} 2^{\frac{k}{2}}\left\|\Delta_{k} u_{0}\right\|_{L^{2}} \lesssim 2^{\frac{k}{2}}\left\|\Delta_{k} u_{0}\right\|_{L^{2}}$,

and consequently, one obtains

$$
\operatorname{supp}_{n}(n+1)^{\frac{1-\varepsilon}{2}}\left\|e^{t \Delta} u_{0}\right\|_{\tilde{L}^{2}\left(n, n+1 ; \dot{\mathcal{B}}_{2,(\infty, 1)}^{\frac{3}{2}}\right)} \lesssim\left\|u_{0}\right\|_{\dot{\mathcal{B}}_{2,(\infty, 1)}^{\frac{1}{2}}}
$$

as desired.

- Second, we focus on the non-homegenous solution $v(t):=\int_{0}^{t} e^{(t-s) \Delta} \mathbb{P} f(s) d s$, where $\mathbb{P}$ is Leray's projection. Fix $t>0$ and let $[t]$ be its integer part. Decompose

$\left\|\Delta_{k} v(t)\right\|_{L_{x}^{2}} \lesssim \sum_{n=0}^{[t]-1} \int_{n}^{n+1} e^{-(t-s) 2^{2 k}}\left\|\Delta_{k} f(s)\right\|_{L^{2}} d s+\int_{[t]}^{t} e^{-(t-s) 2^{2 k}}\left\|\Delta_{k} f(s)\right\|_{L^{2}} d s$.

Notice that if $0 \leq t<1$, then only the last term shows up in the last inequality. We start by estimating $v$ in $\tilde{L}^{\infty}\left(\dot{\mathcal{B}}_{2,(\infty, 1)}^{\frac{1}{2}}\right)$.

For any $n \leq[t]-1$, we have

$$
\begin{aligned}
\int_{n}^{n+1} e^{-(t-s) 2^{2 k}}\left\|\Delta_{k} f(s)\right\|_{L^{2}} d s & \lesssim\left(\max _{n}(n+1)^{\frac{1-\varepsilon}{2}}\left\|\Delta_{k} f\right\|_{L^{2}\left(n, n+1, L_{x}^{2}\right)}\right) \frac{e^{-(t-n-1) 2^{2 k}} \alpha_{k}}{(n+1)^{\frac{1-\varepsilon}{2}} 2^{k}} \\
& \lesssim M_{k, \varepsilon}(f) 2^{-k\left(\frac{1}{2}-\varepsilon\right)} \alpha_{k} e^{-t 2^{2 k}} \frac{e^{(n+1) 2^{2 k}}}{(n+1)^{\frac{1-\varepsilon}{2}}}
\end{aligned}
$$


where we used $\sqrt{1-e^{-22^{2 k}}} \leq \alpha_{k}$ in the first above estimate. Moreover,

$$
\begin{aligned}
\int_{[t]}^{t} e^{-(t-s) 2^{2 k}}\left\|\Delta_{k} f(s)\right\|_{L^{2}} d s & \lesssim\left(\max _{n}(n+1)^{\frac{1-\varepsilon}{2}}\left\|\Delta_{k} f\right\|_{L^{2}\left(n, n+1, L_{x}^{2}\right)}\right) \frac{\sqrt{1-e^{-2(t-[t]) 2^{2 k}}}}{(t+1)^{\frac{1-\varepsilon}{2}} 2^{k}} \\
& \lesssim M_{k, \varepsilon}(f) 2^{-k\left(\frac{1}{2}-\varepsilon\right)} \frac{\min \left(1, \sqrt{2(t-[t]) 2^{2 k}}\right)}{(t+1)^{\frac{1-\varepsilon}{2}}} \\
& \lesssim M_{k, \varepsilon}(f) 2^{-k\left(\frac{1}{2}-\varepsilon\right)} .
\end{aligned}
$$

Thus,

$$
2^{\frac{k}{2}}\left\|\Delta_{k} v(t)\right\|_{L_{x}^{2}} \lesssim M_{k, \varepsilon}(f)\left(\alpha_{k} 2^{k \varepsilon} e^{-t 2^{2 k}} \sum_{n=0}^{[t]-1} \frac{e^{(n+1) 2^{2 k}}}{(n+1)^{\frac{1-\varepsilon}{2}}}+2^{k \varepsilon}\right) .
$$

Now, when $k \leq 0$, to estimate the sum, we distinguish two cases. In the case $[t] 2^{2 k} \leq 1$ we use

$$
\alpha_{k} 2^{k \varepsilon} e^{-t 2^{2 k}} \sum_{n=0}^{[t]-1} \frac{e^{(n+1) 2^{2 k}}}{(n+1)^{\frac{1-\varepsilon}{2}}} \lesssim \alpha_{k} 2^{k \varepsilon}[t]^{\frac{1+\varepsilon}{2}} \lesssim\left(2^{2 k}[t]\right)^{\frac{1+\varepsilon}{2}} \lesssim 1 .
$$

In the case, $[t] 2^{2 k} \geq 1$, we use (3.4) and estimate in the following way

$$
\sum_{n=0}^{[t]-1} \frac{e^{(n+1) 2^{2 k}}}{(n+1)^{\frac{1-\varepsilon}{2}}} \leq[t]^{\frac{\varepsilon}{2}} \sum_{n=0}^{[t]-1} \frac{e^{(n+1) 2^{2 k}}}{\sqrt{n+1}} \lesssim 2^{-k} \frac{e^{[t] 2^{2 k}}}{\left(2^{2 k}[t]\right)^{\frac{1-\varepsilon}{2}}} .
$$

Consequently, in both cases, we have

$$
\alpha_{k} 2^{k \varepsilon} e^{-t 2^{2 k}} \sum_{n=0}^{[t]-1} \frac{e^{(n+1) 2^{2 k}}}{(n+1)^{\frac{1-\varepsilon}{2}}} \lesssim 1
$$

and therefore

$$
\sup _{k \leq 0} \sup _{t>0} 2^{\frac{k}{2}}\left\|\Delta_{k} v(t)\right\|_{L^{2}} \lesssim \sup _{k \leq 0} M_{k, \varepsilon}(f)
$$

Next, we treat frequencies $k \geq 1$. Arguing exactly as above, we obtain

$$
\int_{n}^{n+1} e^{-(t-s) 2^{2 k}}\left\|\Delta_{k} f(s)\right\|_{L^{2}} d s+\int_{[t]}^{t} e^{-(t-s) 2^{2 k}}\left\|\Delta_{k} f(s)\right\|_{L^{2}} d s \lesssim \tilde{M}_{k, \varepsilon}(f) 2^{-\frac{k}{2}}
$$

yielding

$$
\sum_{k \geq 0} \sup _{t>0} 2^{\frac{k}{2}}\left\|\Delta_{k} v(t)\right\|_{L^{2}} \lesssim \sum_{k \geq 0} \sup _{n \in \mathbb{N}}(n+1)^{\frac{1-\varepsilon}{2}} 2^{-\frac{k}{2}}\left\|\Delta_{k} f\right\|_{L^{2}\left(n, n+1 ; L^{2}\right)}
$$


Whence

$$
\operatorname{supp}_{t>0}\|u(t)\|_{\dot{\mathcal{B}}_{2,(\infty, 1)}^{\frac{1}{2}}} \leq\|f\|_{\mathcal{Y}_{1}}
$$

This shows the estimate in $\tilde{L}^{\infty}\left(\dot{\mathcal{B}}_{2,(\infty, 1)}^{\frac{1}{2}}\right)$. Next we will estimate $v(t)$ in $\mathcal{X}_{1}$.

Thanks to (3.9) and (3.10) we have

$$
\begin{aligned}
(t+1)^{\frac{1-\varepsilon}{2}} 2^{k\left(\frac{1}{2}-\varepsilon\right)} \alpha_{k}\left\|\Delta_{k} v(t)\right\|_{L_{x}^{2}} & \lesssim(t+1)^{\frac{1-\varepsilon}{2}} M_{k, \varepsilon}(f) \alpha_{k}^{2} e^{-t 2^{2 k}} \sum_{n=0}^{[t]-1} \frac{e^{(n+1) 2^{2 k}}}{(n+1)^{\frac{1-\varepsilon}{2}}} \\
& +M_{k, \varepsilon}(f) \alpha_{k} .
\end{aligned}
$$

In the case $[t] 2^{2 k} \leq 1$ we use (3.11) to conclude that

$$
(t+1)^{\frac{1-\varepsilon}{2}} M_{k, \varepsilon}(f) \alpha_{k}^{2} e^{-t 2^{2 k}} \sum_{n=0}^{[t]-1} \frac{e^{(n+1) 2^{2 k}}}{(n+1)^{\frac{1-\varepsilon}{2}}} \lesssim M_{k, \varepsilon}(f) \alpha_{k}^{2}(t+1) \lesssim M_{k, \varepsilon}(f) .
$$

In the case $[t] 2^{2 k} \geq 1$ we use (3.12) to conclude that

$$
(t+1)^{\frac{1-\varepsilon}{2}} M_{k, \varepsilon}(f) \alpha_{k}^{2} e^{-t 2^{2 k}} \sum_{n=0}^{[t]-1} \frac{e^{(n+1) 2^{2 k}}}{(n+1)^{\frac{1-\varepsilon}{2}}} \lesssim M_{k, \varepsilon}(f) \alpha_{k}^{2} 2^{k(\varepsilon-2)} \lesssim M_{k, \varepsilon}(f) .
$$

Taking the supremum in $t$ and then the supremum in $k \leq 0$ gives

$$
\operatorname{sũp}_{t>0}(t+1)^{\frac{1-\varepsilon}{2}}\left\|v^{<}(t)\right\|_{\dot{\mathcal{B}}_{2, \infty}^{\frac{3}{2}-\varepsilon}} \lesssim\|f\|_{\mathcal{Y}_{1}} .
$$

Now we consider frequencies $k \geq 1$. We have

$$
\begin{aligned}
(t+1)^{\frac{1-\varepsilon}{2}} 2^{\frac{k}{2}}\left\|\Delta_{k} v(t)\right\|_{L_{x}^{2}} & \lesssim(t+1)^{\frac{1-\varepsilon}{2}} \tilde{M}_{k, \varepsilon}(f) \alpha_{k} e^{-t 2^{2 k}} \sum_{n=0}^{[t]-1} \frac{e^{(n+1) 2^{2 k}}}{(n+1)^{\frac{1-\varepsilon}{2}}} \\
& +M_{k, \varepsilon}(f),
\end{aligned}
$$

and in the case $[t] 2^{2 k} \leq 1$ we use (3.11) to conclude that

$$
(t+1)^{\frac{1-\varepsilon}{2}} \tilde{M}_{k, \varepsilon}(f) e^{-t 2^{2 k}} \sum_{n=0}^{[t]-1} \frac{e^{(n+1) 2^{2 k}}}{(n+1)^{\frac{1-\varepsilon}{2}}} \lesssim \tilde{M}_{k, \varepsilon}(f)(t+1) \lesssim 2^{-2 k} M_{k, \varepsilon}(f) .
$$

In the case $[t] 2^{2 k} \geq 1$ we use (3.12) to conclude that

$$
(t+1)^{\frac{1-\varepsilon}{2}} \tilde{M}_{k, \varepsilon}(f) e^{-t 2^{2 k}} \sum_{n=0}^{[t]-1} \frac{e^{(n+1) 2^{2 k}}}{(n+1)^{\frac{1-\varepsilon}{2}}} \lesssim \tilde{M}_{k, \varepsilon}(f) 2^{-k} \lesssim \tilde{M}_{k, \varepsilon}(f) .
$$


Taking the supremum in $t$ and then summing in $k \geq 0$ gives

$$
\operatorname{sunp}_{t>0}(t+1)^{\frac{1-\varepsilon}{2}}\left\|v^{>}(t)\right\|_{\dot{\mathcal{B}}_{2,1}^{\frac{1}{2}}} \lesssim\|f\|_{\mathcal{Y}_{1}} .
$$

In conclusion, we have shown that

$$
\operatorname{sũp}_{t>0}(t+1)^{\frac{1-\varepsilon}{2}}\|v(t)\|_{\mathcal{B}_{2,(\infty, 1)}^{\frac{3}{2}-\varepsilon, \frac{1}{2}}} \lesssim\|f\|_{\mathcal{Y}_{1}} .
$$

Finally, we estimate $v$ in $\sup _{n}(n+1)^{\frac{1-\varepsilon}{2}}\|v\|_{L^{2}\left(n, n+1 ; \dot{B}_{2,(\infty, 1)}^{\frac{3}{2}}\right)}$. Fix an integer $N$. For all $N \leq t<N+1$, arguing as before we obtain

$$
\begin{aligned}
\left\|\Delta_{k} v(t)\right\|_{L_{x}^{2}} & \lesssim \tilde{M}_{k, \varepsilon}(f) \alpha_{k} 2^{-\frac{k}{2}} \sum_{n=0}^{N-1} \frac{e^{(n+1-t) 2^{2 k}}}{(n+1)^{\frac{1-\varepsilon}{2}}}+\tilde{M}_{k, \varepsilon}(f) \alpha_{k} 2^{-\frac{k}{2}} \frac{\min \left(1, \sqrt{2(t-N) 2^{2 k}}\right)}{(N+1)^{\frac{1-\varepsilon}{2}}} \\
& \lesssim M_{k, 0}(f) \alpha_{k} 2^{-\frac{k}{2}}\left(\int_{0}^{[t]} \frac{e^{-(t-s) 2^{2 k}}}{\sqrt{s}} d s+\frac{e^{(N-t) 2^{2 k}}}{\sqrt{N+1}}\right) \\
& \lesssim M_{k, 0}(f) \alpha_{k} 2^{-\frac{k}{2}}\left(1+\frac{e^{(N-t) 2^{2 k}}}{\sqrt{N+1}}\right)
\end{aligned}
$$

and

$$
\left\|\Delta_{k} v(t)\right\|_{L^{2}\left(N, N+1, L_{x}^{2}\right)} \lesssim \tilde{M}_{k, \varepsilon}(f) \alpha_{k}^{2} 2^{-\frac{3}{2} k} \sum_{n=0}^{N-1} \frac{e^{(n+1-N) 2^{2 k}}}{(n+1)^{\frac{1-\varepsilon}{2}}}
$$

In the case $[t] 2^{2 k} \leq 1$, we control the sum using (3.11) to end up with

$$
\left\|\Delta_{k} v(t)\right\|_{L^{2}\left(N, N+1, L_{x}^{2}\right)} \lesssim 2^{-\frac{3}{2} k} \tilde{M}_{k, \varepsilon}(f) \alpha_{k}^{2} N^{\frac{1+\varepsilon}{2}} .
$$

This leads to

$$
(N+1)^{\frac{1+\varepsilon}{2}} 2^{\frac{3}{2} k}\left\|\Delta_{k} v(t)\right\|_{L^{2}\left(N, N+1, L_{x}^{2}\right)} \lesssim \tilde{M}_{k, \varepsilon}(f) \alpha_{k}^{2} N \lesssim \tilde{M}_{k, \varepsilon}(f)
$$

In the case $[t] 2^{2 k} \geq 1$, we estimate the sum using (3.12) to obtain

$$
\left\|\Delta_{k} v(t)\right\|_{L^{2}\left(N, N+1, L_{x}^{2}\right)} \lesssim 2^{-\frac{3}{2} k} \tilde{M}_{k, \varepsilon}(f) \alpha_{k}^{2} \frac{2^{-k}}{\left(2^{2 k} N\right)^{\frac{1-\varepsilon}{2}}}
$$

yielding estimates

$$
(N+1)^{\frac{1-\varepsilon}{2}} 2^{\frac{3}{2} k}\left\|\Delta_{k} v(t)\right\|_{L^{2}\left(N, N+1, L_{x}^{2}\right)} \lesssim \tilde{M}_{k, \varepsilon}(f) \alpha_{k}^{2} 2^{(\varepsilon-2) k} \lesssim \tilde{M}_{k, \varepsilon}(f),
$$


and

$$
\operatorname{sũp}_{N}(N+1)^{\frac{1-\varepsilon}{2}}\|v(t)\|_{\tilde{L}^{2}\left(N, N+1 ; \dot{\mathcal{B}}_{2,(\infty, 1)}^{\frac{3}{2}}\right)} \lesssim\|f\|_{\mathcal{Y}_{1}}
$$

as desired. This completes the proof of the Lemma.

Now we focus on Maxwell's equations. The following result quantifies a weak form of decay for the electromagnetic field $(E, B)$.

Lemma 3.2. Let $G \in \mathcal{Y}_{1}$, and $(E, B)$ be a smooth solution of

$$
\left\{\begin{array}{l}
\partial_{t} E-\nabla \wedge B+E=G \\
\partial_{t} B+\nabla \wedge E=0 \\
(E, B)_{\mid t=0}=\left(E^{0}, B^{0}\right)
\end{array}\right.
$$

on some time interval $[0, T]$. Then, the following estimate holds (with constants independent of $T$ )

$$
\|E\|_{\mathcal{X}_{2}}+\|B\|_{\mathcal{X}_{3}} \lesssim\left\|\left(E^{0}, B^{0}\right)\right\|_{H^{\frac{1}{2}}}+\|G\|_{\mathcal{Y}_{2}}
$$

Proof. First, we recall that in [1], the following estimate was proven.

$$
\|E\|_{\tilde{L}_{T}^{\infty} H^{\frac{1}{2}} \cap L_{T}^{2} \dot{H}^{\frac{1}{2}}}+\|B\|_{\tilde{L}_{T}^{\infty} H^{\frac{1}{2}} \cap L_{T}^{2} \dot{H}^{1, \frac{1}{2}}} \lesssim\left\|\left(E_{0}, B_{0}\right)\right\|_{H^{\frac{1}{2}}}+\|G\|_{L_{T}^{2} H^{\frac{1}{2}}}
$$

with a constant independent of $T$. Hence, it will be sufficient to prove that

$$
\operatorname{sũp}_{t>0} t^{\frac{1}{2}}\|E(t)\|_{\dot{H}^{\frac{1}{2}}}+\operatorname{sũp}_{t>0} t^{\frac{1}{2}}\|B(t)\|_{\dot{H}^{1, \frac{1}{2}}} \lesssim\left\|\left(E_{0}, B_{0}\right)\right\|_{H^{\frac{1}{2}}}+\|G\|_{L_{T}^{2} H^{\frac{1}{2}}} \cdot
$$

We decompose $E=E_{\sigma}+E_{\nabla}$ into its divergence free component $E_{\sigma}$, and irrotational component $E_{\nabla}$. It is easy to check that $B, E_{\sigma}$ and $E_{\nabla}$ solve

$$
\begin{gathered}
\partial_{t}^{2} B-\Delta B+\partial_{t} B=-\nabla \times G, \\
\partial_{t} B+\nabla \times E_{\sigma}=0
\end{gathered}
$$

and

$$
\partial_{t} E_{\nabla}+E_{\nabla}=G_{\nabla}
$$

respectively. Thanks to the Fourier transform, and the spectral analysis given in the Appendix we have the following representation formula for $B$.

$$
\hat{B}(t)=e^{t \lambda_{-}} \hat{B}^{0}+\left(e^{t \lambda_{+}}-e^{t \lambda_{-}}\right) b^{0}+\frac{i}{\lambda_{-}} \int_{0}^{t}\left(e^{(t-\tau) \lambda_{+}}-e^{(t-\tau) \lambda_{-}}\right) \xi \times e d \tau,
$$


where,

$$
\lambda_{ \pm}(\xi)=\frac{-1 \pm \sqrt{1-4|\xi|^{2}}}{2}
$$

the initial data $\left(\hat{E}^{0}, \hat{B}^{0}\right)$ and the source term $\nabla \wedge G$ are decomposed as follows

$$
\left(\begin{array}{c}
\hat{E}^{0} \\
\hat{B}^{0}
\end{array}\right)=\left(\begin{array}{c}
\frac{\xi \cdot \hat{E}^{0}}{|\xi|^{2}} \xi \\
0
\end{array}\right)+\left(\begin{array}{c}
e^{0} \\
\frac{-i}{\lambda_{-}} \xi \times e^{0}
\end{array}\right)+\left(\begin{array}{c}
\frac{-i}{\lambda_{-}} \xi \times b^{0} \\
b^{0}
\end{array}\right),
$$

with $\xi \cdot e^{0}=\xi \cdot b^{0}=0$, and

$$
\left(\begin{array}{c}
\hat{G} \\
0
\end{array}\right)=\left(\begin{array}{c}
\frac{\xi \cdot \hat{G}}{|\xi|^{2}} \xi \\
0
\end{array}\right)+\left(\begin{array}{c}
e \\
\frac{-i}{\lambda_{-}} \xi \times e
\end{array}\right)+\left(\begin{array}{c}
\frac{-i}{\lambda_{-}} \xi \times b \\
b
\end{array}\right),
$$

with $\xi \cdot e=\xi \cdot b=0$.

Let $K$ be some fixed parameter $1<K<2$ determined such that for all $|\xi| \geq \frac{1}{2 K}$ we have $\mathcal{R}\left(\lambda_{ \pm}\right)<-\frac{1}{4}$. To this end, we further decompose the magnetic fields as follows.

$$
B=B_{<}+B_{>}
$$

where, for

$$
\begin{aligned}
& \hat{B}_{<}=\mathbb{1}_{\left\{|\xi| \leq \frac{1}{2 K}\right\}} \hat{B}, \\
& \hat{B}_{>}=\mathbb{1}_{\left\{\frac{1}{2 K}<|\xi|\right\}} \hat{B} .
\end{aligned}
$$

The first corresponds to the low frequency component of $B$ and the second to the high frequency. Now we estimate each of the above terms separately. Thanks to (3.4), we have

$$
\begin{aligned}
t^{\frac{1}{2}} 2^{\frac{k}{2}}\left\|\Delta_{k} B_{>}(t)\right\|_{L^{2}} & \lesssim t^{\frac{1}{2}} 2^{\frac{k}{2}} e^{-\frac{t}{4}}\left(\left\|\Delta_{k} E^{0}\right\|_{L^{2}}+\left\|\Delta_{k} B^{0}\right\|_{L^{2}}\right) \\
& +t^{\frac{1}{2}} \int_{0}^{t} e^{-\frac{t-s}{4}} 2^{\frac{k}{2}}\left\|\Delta_{k} G(s)\right\|_{L^{2}} d s \\
& \lesssim 2^{\frac{k}{2}}\left(\left\|\Delta_{k} E^{0}\right\|_{L^{2}}+\left\|\Delta_{k} B^{0}\right\|_{L^{2}}\right) \\
& +\sup _{t>0}\left(t^{\frac{1}{2}} 2^{\frac{k}{2}}\left\|\Delta_{k} G(t)\right\|_{L^{2}}\right) t^{\frac{1}{2}} e^{-\frac{t}{4}} \int_{0}^{t} \frac{e^{\frac{s}{4}}}{\sqrt{s}} d s
\end{aligned}
$$

Again, using (3.4) we conclude that

$$
\begin{aligned}
t^{\frac{1}{2}} 2^{\frac{k}{2}}\left\|\Delta_{k} B_{>}(t)\right\|_{L^{2}} & \lesssim \sup _{t>0}\left(t^{\frac{1}{2}} 2^{\frac{k}{2}}\left\|\Delta_{k} G(t)\right\|_{L^{2}}\right) \\
& +2^{\frac{k}{2}}\left(\left\|\Delta_{k} E^{0}\right\|_{L^{2}}+\left\|\Delta_{k} B^{0}\right\|_{L^{2}}\right) .
\end{aligned}
$$

Now we estimate $B_{<}$. From Duhamel's formula, Lemma A.1 and (3.4) we estimate 


$$
\begin{aligned}
t^{\frac{1}{2}} 2^{k}\left\|\Delta_{k} B_{<}(t)\right\|_{L^{2}} & \lesssim t^{\frac{1}{2}} 2^{k}\left(e^{-\frac{t}{2}}\left\|\Delta_{k}\left(B^{0}-b^{0}\right)\right\|_{L^{2}}+e^{-t 2^{k}}\left\|\Delta_{k} b^{0}\right\|_{L^{2}}\right) \\
& +t^{\frac{1}{2}} 2^{k} \int_{0}^{t} e^{-(t-s) 2^{2 k}}\left\|\Delta_{k} G_{<}(s)\right\|_{L^{2}} d s \\
& +t^{\frac{1}{2}} 2^{k} \int_{0}^{t} e^{-\frac{t-s}{2}}\left\|\Delta_{k} G_{<}(s)\right\|_{L^{2}} d s \\
& \lesssim 2^{k}\left\|\Delta_{k}\left(B^{0}-b^{0}\right)_{<}\right\|_{L^{2}}+\left\|\Delta_{k} b_{<}^{0}\right\|_{L^{2}}+\operatorname{sunp}_{t>0} t^{\frac{1}{2}}\left\|\Delta_{k} G_{<}(t)\right\|_{L_{x}^{2}}
\end{aligned}
$$

which, in virtue of Lemma A.1 and the identity

$$
\hat{B}^{0}-b^{0}=-\frac{i}{\lambda_{-}} \xi \times e^{0} .
$$

gives

$$
\begin{aligned}
t^{\frac{1}{2}} 2^{k}\left\|\Delta_{k} B_{<}(t)\right\|_{L^{2}} & \lesssim\left\|\Delta_{k}\left(B^{0}-b^{0}\right)<\right\|_{L^{2}}+\left\|\Delta_{k} b_{<}^{0}\right\|_{L^{2}} \\
& +\operatorname{sunpt}_{t>0} t^{\frac{1}{2}}\left\|\Delta_{k} G_{<}(t)\right\|_{L_{x}^{2}} .
\end{aligned}
$$

Taking the $\ell^{2}$ summation in $k$ gives

$$
\operatorname{sũp}_{t>0} t^{\frac{1}{2}}\left\|B_{<}\right\|_{\dot{H}^{1}} \lesssim\left\|\left(E^{0}, B^{0}\right)\right\|_{L^{2}}+\operatorname{supp}_{t>0} t^{\frac{1}{2}}\|G\|_{L_{x}^{2}},
$$

as desired. To estimate $E$, it is sufficient to estimate

$$
\operatorname{supp}_{t>0} t^{\frac{1}{2}}\left\|E_{\nabla}\right\|_{H^{\frac{1}{2}}}, \quad \text { and } \operatorname{sunp}_{t>0} t^{\frac{1}{2}}\left\|\nabla \times E_{\sigma}\right\|_{\dot{H}^{-1,-\frac{1}{2}}}
$$

because

$$
\operatorname{sũp}_{t>0} t^{\frac{1}{2}}\left\|E_{\sigma}\right\|_{H^{\frac{1}{2}}} \lesssim \operatorname{sũp}_{t>0} t^{\frac{1}{2}}\left\|\nabla \times E_{\sigma}\right\|_{\dot{H}^{-1,-\frac{1}{2}}} .
$$

Thanks to Faraday's law, we have

$$
\left\|\nabla \times E_{\sigma}\right\|_{\dot{H}^{-1,-\frac{1}{2}}}=\left\|\partial_{t} B\right\|_{\dot{H}^{-1,-\frac{1}{2}}} .
$$

From Duhamel's formula, we have

$$
\partial_{t} \hat{B}=\lambda_{-} e^{t \lambda_{-}} \hat{B}^{0}+\left(\lambda_{+} e^{t \lambda_{+}}-\lambda_{-} e^{t \lambda_{-}}\right) b^{0}+\frac{i}{\lambda_{-}} \int_{0}^{t}\left(\lambda_{+} e^{(t-s) \lambda_{+}}-\lambda_{-} e^{(t-s) \lambda_{-}}\right) \xi \times e d s .
$$


Using Lemma A.1, we have for all $k \leq 1$,

$$
\begin{aligned}
t^{\frac{1}{2}} 2^{-k}\left\|\Delta_{k}\left(\nabla \times E_{\sigma}\right)\right\|_{L^{2}} & \lesssim t^{\frac{1}{2}} 2^{-k} 2^{2 k} e^{-t 2^{2 k}}\left\|\Delta_{k} b^{0}\right\|_{L^{2}}+t^{\frac{1}{2}} 2^{-k} e^{-\frac{t}{2}}\left\|\Delta_{k}\left(B^{0}-b^{0}\right)\right\|_{L^{2}} \\
& +t^{\frac{1}{2}} 2^{2 k} \int_{0}^{t} e^{(s-t) 2^{2 k}}\left\|\Delta_{k} e(s)\right\|_{L^{2}} d s+t^{\frac{1}{2}} \int_{0}^{t} e^{\frac{s-t}{2}}\left\|\Delta_{k} e(s)\right\|_{L^{2}} d s \\
& \lesssim\left\|\Delta_{k} b^{0}\right\|_{L^{2}}+\left(\left\|\Delta_{k} E^{0}\right\|_{L^{2}}+\left\|\Delta_{k} B^{0}\right\|_{L^{2}}\right) \\
& +\sup _{t>0}\left(t^{\frac{1}{2}}\left\|\Delta_{k} G\right\|_{L^{2}}\right) t^{\frac{1}{2}} 2^{2 k} e^{-t 2^{2 k}} \int_{0}^{t} \frac{e^{-s 2^{2 k}}}{\sqrt{s}} d s \\
& +\sup _{t>0}\left(t^{\frac{1}{2}}\left\|\Delta_{k} G\right\|_{L^{2}}\right) t^{\frac{1}{2}} e^{-\frac{t}{2}} \int_{0}^{t} \frac{e^{\frac{s}{2}}}{\sqrt{s}} d s .
\end{aligned}
$$

Again, it is important to mention that in the second estimate in above we used (3.24). Now for $k \geq 2$

$$
\begin{aligned}
t^{\frac{1}{2}} 2^{-\frac{k}{2}}\left\|\Delta_{k}\left(\nabla \times E_{\sigma}\right)\right\|_{L^{2}} & \lesssim t^{\frac{1}{2}} 2^{\frac{k}{2}} e^{-\frac{t}{2}}\left(\left\|\Delta_{k} E^{0}\right\|_{L^{2}}+\left\|\Delta_{k} B^{0}\right\|_{L^{2}}\right) \\
& +t^{\frac{1}{2}} 2^{\frac{k}{2}} \int_{0}^{t} e^{(s-t) 2^{2 k}}\left\|\Delta_{k} G\right\|_{L^{2}} d s \\
& \lesssim 2^{\frac{k}{2}}\left(\left\|\Delta_{k} E^{0}\right\|_{L^{2}}+\left\|\Delta_{k} B^{0}\right\|_{L^{2}}\right) \\
& +\sup _{t>0}\left(t^{\frac{1}{2}} 2^{\frac{k}{2}}\left\|\Delta_{k} G\right\|_{L^{2}}\right) t^{\frac{1}{2}} e^{-t 2^{2 k}} \int_{0}^{t} e^{s 2^{2 k}} \frac{d s}{\sqrt{s}} .
\end{aligned}
$$

Using (3.4) and taking the $\ell^{2}$ summation concludes the proof. To estimate $\sup _{t>0} t^{\frac{1}{2}}\left\|E_{\nabla}\right\|_{H^{\frac{1}{2}}}$, we also write Duhamel's formula for $E_{\nabla}$.

$$
E_{\nabla}=e^{-t} E_{\nabla}^{0}+\int_{0}^{t} e^{s-t} G_{\nabla}(s) d s
$$

Then,

$t^{\frac{1}{2}} 2^{\frac{k}{2}}\left\|\Delta_{k} E_{\nabla}\right\|_{L^{2}} \lesssim t^{\frac{1}{2}} 2^{\frac{k}{2}} e^{-t}\left\|\Delta_{k} E_{\nabla}^{0}\right\|_{L^{2}}+t^{\frac{1}{2}} e^{-t} \sup _{t>0}\left(t^{\frac{1}{2}} 2^{\frac{k}{2}}\left\|\Delta_{k} G_{\nabla}\right\|_{L^{2}}\right) \int_{0}^{t} \frac{e^{s}}{\sqrt{s}} d s$

which finishes the proof of the Lemma.

\subsection{Nonlinear estimates}

The following is a series of nonlinear estimates needed for the contraction argument. The first Lemma is essential to estimate the nonlinearity in Maxwell-Ampère's equation.

Lemma 3.3. For all smooth functions $u, E$ and $B$ defined on some interval $[0, T]$, we have the following estimates, with constants independent of $T$ : 


$$
\begin{gathered}
\|u \wedge B\|_{\mathcal{Y}_{2}} \lesssim\|u\|_{\tilde{L}^{2}\left(\dot{\mathcal{B}}_{2,(\infty, 1)}^{\frac{3}{2}}\right) \cap \sup _{\mathrm{t}} t^{\frac{1}{2}} \dot{\mathcal{B}}_{2,(\infty, 1)}^{\frac{3}{2}}}\|B\|_{\mathcal{X}_{3}} \\
\left\|u_{p e r} \wedge B\right\|_{\mathcal{Y}_{2}} \lesssim\left\|u_{p e r}\right\|_{\tilde{L}_{p e r}^{2}\left(\dot{\mathcal{B}}_{2,(\infty, 1)}^{\frac{3}{2}}\right) \cap \tilde{L}^{\infty}\left(\dot{\mathcal{B}}_{2,(\infty, 1)}^{\frac{1}{2}}\right)}\|B\|_{\mathcal{X}_{3}} \\
\left\|u \wedge B_{p e r}\right\| \mathcal{Y}_{2} \lesssim\|u\| \mathcal{X}\left\|B_{p e r}\right\|_{\tilde{L}_{p e r}^{\infty}\left(H^{\frac{1}{2}}\right)}
\end{gathered}
$$

The second Lemma is to estimate bilinear terms in the Navier-Stokes equations

\section{Lemma 3.4.}

$$
\begin{aligned}
& \|\nabla(u \otimes v)\|_{\mathcal{Y}_{1}} \lesssim\|u\|_{\mathcal{X}}\|v\|_{\mathcal{X}} \\
& \left\|\nabla\left(u_{p e r} \otimes v\right)\right\|\left\|_{\mathcal{Y}_{1}} \lesssim\right\| v\left\|_{\mathcal{X}_{1}}\right\| u_{p e r} \|_{\tilde{L}_{p e r}^{\infty}\left(\dot{\mathcal{B}}_{2,(\infty, 1)}^{\frac{1}{2}}\right)} \\
& \left\|E_{\text {per }} \wedge B\right\|_{\mathcal{Y}_{1}} \lesssim\left\|E_{\text {per }}\right\|_{\tilde{L}^{\infty}\left(H^{\frac{1}{2}}\right)}\|B\|_{\mathcal{X}_{3}}, \\
& \left\|E \wedge B_{p e r}\right\|\left\|_{\mathcal{Y}_{1}} \lesssim\right\| E\left\|_{\mathcal{X}_{2}}\right\| B_{p e r} \|_{\tilde{L}_{p e r}^{\infty}\left(H^{\frac{1}{2}}\right)}, \\
& \|E \wedge B\|_{\mathcal{Y}_{1}} \lesssim\|E\|_{\mathcal{X}_{2}}\|B\|_{\tilde{L}^{\infty} H^{\frac{1}{2}}}
\end{aligned}
$$

The last Lemma gives the estimates of the trilinear terms in the equation of the velocity vector field.

\section{Lemma 3.5.}

$$
\begin{aligned}
& \left\|\left(u \wedge B_{p e r}\right) \wedge B_{p e r}\right\|_{\mathcal{Y}_{1}} \lesssim\|u\|_{\mathcal{X}}\left\|B_{p e r}\right\|_{\tilde{L}^{\infty}\left(H^{\frac{1}{2}}\right)}^{2} \\
& \left\|(u \wedge B) \wedge B_{p e r}\right\|\left\|_{\mathcal{Y}_{1}} \lesssim\right\| u\left\|_{\mathcal{X}}\right\| B\left\|_{L^{\infty}\left(H^{\frac{1}{2}}\right)}\right\| B_{p e r} \|_{\tilde{L}^{\infty}\left(H^{\frac{1}{2}}\right)} \\
& \left\|\left(u_{p e r} \wedge B\right) \wedge B\right\|\left\|_{\mathcal{Y}_{1}} \lesssim\right\| u_{\text {per }}\left\|_{\tilde{L}_{p e r}^{\infty}\left(\dot{\mathcal{B}}_{2,1}^{\frac{1}{2}}\right)}\right\| B\left\|_{\mathcal{X}_{3}}\right\| B \|_{\mathcal{X}_{3}} \\
& \left\|\left(u_{p e r} \wedge B\right) \wedge B_{p e r}\right\|\left\|_{\mathcal{Y}_{1}} \lesssim\right\| u_{p e r}\left\|_{\tilde{L}_{p e r}^{\infty}\left(\dot{\mathcal{B}}_{2,(\infty, 1)}^{\frac{1}{2}}\right) \cap \tilde{L}_{p e r}^{2}\left(\dot{\mathcal{B}}_{2,(\infty, 1)}^{\frac{3}{2}}\right)}\right\| B_{p e r}\left\|_{\tilde{L}^{\infty}\left(H^{\frac{1}{2}}\right)}\right\| B \| \chi_{\left.\aleph_{3}^{3} .36\right)}
\end{aligned}
$$


Proof. We only show how to estimate the worst terms $\left(u_{\text {per }} \wedge B_{\text {per }}\right) \wedge B$ and $\left(u \wedge B_{\text {per }}\right) \wedge B_{\text {per }}$ because all the other estimates are easier. It is enough to show the following

$$
\sup _{n}(1+n)^{\frac{1-\varepsilon}{2}}\left\|u_{\mathrm{per}} \wedge B\right\|_{L^{2}\left(n, n+1 ; H^{\frac{1}{2}}\right)} \lesssim\left\|u_{\mathrm{per}}\right\|\left\|_{\mathcal{X}_{1}}\right\| B \|_{\mathcal{X}_{3}}
$$

and

$$
\sup _{n}(1+n)^{\frac{1-\varepsilon}{2}}\left\|B_{\text {per }} \wedge F\right\|_{\mathcal{Y}_{1}} \lesssim\left\|B_{\text {per }}\right\|_{\tilde{L}^{\infty}\left(H^{\frac{1}{2}}\right)} \sup _{n}(1+n)^{\frac{1-\varepsilon}{2}}\|F\|_{L^{2}\left(n, n+1 ; H^{\frac{1}{2}}\right)}
$$

for $F=u \wedge B_{\text {per }}$.

We begin by proving (3.37), and estimate the term $T_{u_{\mathrm{per}}} B$ in the paraproduct. First, as in the proof of Lemma 2.3, we have

$$
\begin{aligned}
\sup _{n}(1+n)^{\frac{1-\varepsilon}{2}}\left\|T_{u_{\mathrm{per}}} B\right\|_{L^{2}\left(n, n+1 ; H^{\frac{1}{2}}\right)} & \lesssim\left\|u_{\mathrm{per}}\right\|_{L_{\mathrm{per}}^{\infty}\left(L^{\infty}\right)} \operatorname{sunp}_{t}(1+t)^{\frac{1-\varepsilon}{2}}\|B\|_{H^{\frac{1}{2}}} \\
& \lesssim\left\|u_{\mathrm{per}}\right\| \mathcal{X}_{1} \operatorname{sunp}_{t}(1+t)^{\frac{1-\varepsilon}{2}}\|B\|_{H^{\frac{1}{2}}} .
\end{aligned}
$$

Next, for $k \leq 0$, by Bernstein's lemma we have

$\sum_{j \leq k} 2^{j}\left\|\Delta_{j} B\right\|_{L^{2}} 2^{\frac{j-k}{2}} 2^{k}\left\|\Delta_{k} u_{\mathrm{per}}\right\|_{L^{2}} \leq \sum_{j \leq k} 2^{j}\left\|\Delta_{j} B\right\|_{L^{2}} 2^{\frac{j-k}{2}}\left\|u_{\mathrm{per}}\right\|_{\tilde{L}_{\mathrm{per}}^{\infty}\left(\dot{B}_{2,(\infty, 1)}^{\frac{1}{2}}\right)}$

while for $k \geq 1$, we decompose further as follows

$$
\begin{aligned}
\sum_{j \leq k} 2^{\frac{3 j}{2}}\left\|\Delta_{j} B\right\|_{L^{2}} 2^{\frac{k}{2}}\left\|\Delta_{k} u\right\|_{L^{2}} & \leq \sum_{j \leq 0} 2^{j}\left\|\Delta_{j} B\right\|_{L^{2}} 2^{\frac{j}{2}} 2^{\frac{k}{2}}\left\|\Delta_{k} u_{\mathrm{per}}\right\|_{L^{2}} \\
& +\sum_{0 \leq j \leq k} 2^{\frac{j}{2}}\left\|\Delta_{j} B\right\|_{L^{2}} 2^{j-k} 2^{\frac{3 k}{2}}\left\|\Delta_{k} u_{\mathrm{per}}\right\|_{L^{2}}
\end{aligned}
$$

which thanks to Young's inequality and after integration in time give

$$
\begin{aligned}
\sup _{n}(1+n)^{\frac{1-\varepsilon}{2}}\left\|T_{B} u_{\text {per }}\right\|_{L^{2}\left(n, n+1 ; H^{\frac{1}{2}}\right)} & \lesssim\left\|u_{\text {per }}\right\|_{\tilde{L}_{\text {per }}^{\infty}\left(\dot{B}_{2,(\infty, 1)}^{\frac{1}{2}}\right)} \sup _{n}(1+n)^{\frac{1-\varepsilon}{2}}\|B\|_{L^{2}\left(n, n+1 ; \dot{H}^{1, \frac{1}{2}}\right)} \\
& +\left\|u_{\mathrm{per}}\right\|_{\tilde{L}_{\mathrm{per}}^{\infty}\left(\dot{B}_{2,(\infty, 1)}^{\frac{3}{2}}\right)} \operatorname{sũp}_{t}(1+t)^{\frac{1-\varepsilon}{2}}\|B\|_{H^{\frac{1}{2}}} \cdot
\end{aligned}
$$

Thus

$$
\sup _{n}(1+n)^{\frac{1-\varepsilon}{2}}\left\|T_{B} u_{\text {per }}\right\|_{L^{2}\left(n, n+1 ; H^{\frac{1}{2}}\right)} \lesssim\left\|u_{\text {per }}\right\| \mathcal{X}_{1}\|B\|_{\mathcal{X}_{3}} .
$$

Finally we estimate the rest $R\left(u_{\text {per }}, B\right)$ only when $k \leq 0$ because the case $k \geq 1$ is easier. By Bernstein's lemma we have

$$
\begin{aligned}
2^{\frac{k}{2}} \sum_{j \geq k-2} 2^{\frac{3 k}{2}}\left\|\Delta_{j} B\right\|_{L^{2}}\left\|\Delta_{j} u_{\mathrm{per}}\right\|_{L^{2}} & \leq 2^{2 k}\left(\sum_{j \leq 0}(\cdot)+\sum_{k-2 \leq j \leq 0}(\cdot)\right) \\
& \lesssim\left\|u_{\mathrm{per}}\right\|_{\dot{B}_{2,(\infty, 1)}^{\frac{1}{2}}\|B\|_{L^{2}} 2^{2 k}} \\
& +\sum_{j \geq k-2} 2^{\frac{3 j}{2}\left\|\Delta_{j} u_{\mathrm{per}}\right\|_{L^{2}} 2^{\frac{j}{2}}\left\|\Delta_{j} B\right\|_{L^{2}} 2^{2(k-j)} .}
\end{aligned}
$$


This concludes that

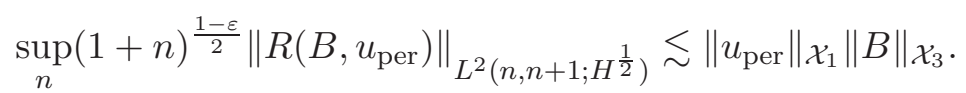

Now we show (3.38). For $k \leq 0$, by Bernstein's lemma we have $2^{-k\left(\frac{1}{2}+\varepsilon\right)}\left\|\Delta_{k} T_{B_{\mathrm{per}}} F\right\|_{L^{2}} \leq 2^{\frac{k}{2}}\left\|\Delta_{k} F\right\|_{L^{2}} \sum_{j \leq k} 2^{j\left(\frac{1}{2}-\varepsilon\right)}\left\|\Delta_{j} B_{\mathrm{per}}\right\|_{L^{2}} 2^{(j-k)(1+\varepsilon)}$.

Additionally, when $k \geq 1$, we estimate as follows

$$
\begin{aligned}
& 2^{-\frac{k}{2}}\left\|\Delta_{k} T_{B_{\mathrm{per}}} F\right\|_{L^{2}} \lesssim 2^{\frac{k}{2}}\left\|\Delta_{k} F\right\|_{L^{2}}\left(\left\|B_{\mathrm{per}}\right\|_{L^{2}}+\sum_{0 \leq j \leq k} 2^{\frac{j}{2}}\left\|\Delta_{j} B_{\mathrm{per}}\right\|_{L^{2}} 2^{j-k}\right) \\
& \lesssim 2^{\frac{k}{2}}\left\|\Delta_{k} F\right\|_{L^{2}}\left\|B_{\text {per }}\right\|_{H^{\frac{1}{2}}} \cdot
\end{aligned}
$$

The last two estimates combined give

$$
\left.\sup _{n}(1+n)^{\frac{1-\varepsilon}{2}} \| T_{B_{\mathrm{per}}} F\right)\left\|_{L^{2}\left(n, n+1 ; \dot{B}_{2,(\infty, 1)}^{\left.-\frac{1}{2},-\frac{1}{2}\right)}\right.} \lesssim \sup _{n}(1+n)^{\frac{1-\varepsilon}{2}}\right\| F\left\|_{L^{2}\left(n, n+1 ; H^{\frac{1}{2}}\right)}\right\| B_{\mathrm{per}} \|_{\tilde{L}^{\infty}\left(H^{\frac{1}{2}}\right)^{.}}
$$

Since the force $F$ and the magnetic field $B$ share the same space regularity, then the same analysis in above implies

$$
\left.\sup _{n}(1+n)^{\frac{1-\varepsilon}{2}} \| T_{F} B_{\text {per }}\right)\left\|_{L^{2}\left(n, n+1 ; \dot{B}_{2,(\infty, 1)}^{\left.-\frac{1}{2},-\frac{1}{2}\right)}\right.} \lesssim \sup _{n}(1+n)^{\frac{1-\varepsilon}{2}}\right\| F\left\|_{L^{2}\left(n, n+1 ; H^{\frac{1}{2}}\right)}\right\| B_{\text {per }} \|_{\tilde{L}^{\infty}\left(H^{\frac{1}{2}}\right)} .
$$

Finally the remaining term is estimated thanks to the following observation

$$
2^{-k\left(\frac{1}{2}+\varepsilon\right)} \sum_{j \geq k-2} 2^{\frac{3 k}{2}}\left\|\Delta_{j} B_{\mathrm{per}}\right\|_{L^{2}}\left\|\Delta_{j} F\right\|_{L^{2}} \lesssim \sum_{j \geq k-2} 2^{j\left(\frac{1}{2}-\varepsilon\right)}\left\|\Delta_{j} B_{\mathrm{per}}\right\|_{L^{2}} 2^{\frac{j}{2}}\left\|\Delta_{j} F\right\|_{L^{2}} 2^{(k-j)(1-\varepsilon)} .
$$

Now we move to the term $\left(u \wedge B_{\text {per }}\right) \wedge B_{\text {per }}$, and begin by the recalling that

$$
H^{\frac{1}{2}} \times H^{\frac{1}{2}} \hookrightarrow B_{2,1}^{-\frac{1}{2}}\left(\mathbb{R}^{3}\right)
$$

Hence, $F:=B_{\text {per }} \cdot B_{\text {per }}$ belongs to $B_{2,1}^{-\frac{1}{2}}\left(\mathbb{R}^{3}\right)$. Next we use again para-product to estimate $F \wedge u$. For $k \leq 0$, by Bernstein's lemma we have

$$
2^{-k\left(\frac{1}{2}+\varepsilon\right)}\left\|\Delta_{k} T_{u} F\right\|_{L^{2}} \leq 2^{-\frac{k}{2}}\left\|\Delta_{k} F\right\|_{L^{2}} \sum_{j \leq k} 2^{j\left(\frac{3}{2}-\varepsilon\right)}\left\|\Delta_{j} u\right\|_{L^{2}} 2^{(j-k) \varepsilon},
$$

and, similarly, when $k \geq 1$ we estimate as follows

$$
2^{-\frac{k}{2}}\left\|\Delta_{k} T_{u} F\right\|_{L^{2}} \lesssim 2^{-\frac{k}{2}}\left\|\Delta_{k} F\right\|_{L^{2}}\left(\sum_{j \leq 0} 2^{j\left(\frac{3}{2}-\varepsilon\right)}\left\|\Delta_{j} u\right\|_{L^{2}} 2^{j \varepsilon}+\sum_{0 \leq j \leq k} 2^{\frac{3 j}{2}}\left\|\Delta_{j} u\right\|_{L^{2}}\right)
$$


giving

$$
2^{-\frac{k}{2}}\left\|\Delta_{k} T_{u} F\right\|_{L^{2}\left(n, n+1 ; L^{2}\right)} \lesssim 2^{-\frac{k}{2}}\left\|\Delta_{k} F\right\|_{L^{2}}\|u\|_{\tilde{L}^{2}\left(n, n+1 ; \dot{B}_{2,(\infty, 1)}^{\frac{3}{2}-\varepsilon, \frac{3}{2}}\right.}
$$

Finally, for $k \leq 0$, we have

$$
2^{-k\left(\frac{1}{2}+\varepsilon\right)}\left\|\Delta_{k} T_{F} u\right\|_{L^{2}} \leq 2^{k\left(\frac{3}{2}-\varepsilon\right)}\left\|\Delta_{k} u\right\|_{L^{2}} \sum_{j \leq k} 2^{-\frac{j}{2}}\left\|\Delta_{j} F\right\|_{L^{2}} 2^{2(j-k)},
$$

and, similarly, when $k \geq 1$ we have

$2^{-\frac{k}{2}}\left\|\Delta_{k} T_{u} F\right\|_{L^{2}} \lesssim 2^{\frac{3 k}{2}}\left\|\Delta_{k} u\right\|_{L^{2}}\left(2^{-2 k}\|F\|_{\dot{B}_{2,1}^{-\frac{1}{2}}}+\sum_{0 \leq j \leq k} 2^{-\frac{j}{2}}\left\|\Delta_{j} F\right\|_{L^{2}} 2^{2(j-k)}.\right)$

Putting together (3.41), (3.41), (3.41) and (3.42) finishes the proof of the Lemma.

\subsection{End of the proof of Theorem 1.3}

Proof. of the Proposition 3.1 First, notice that $\Phi\left(-e^{t \mathcal{A}} \Gamma_{\text {err }}^{0}\right)=0$, while by Lemma 3.1 and Lemma 3.2, we have

$$
\left\|e^{t \mathcal{A}} \Gamma_{\text {err }}^{0}\right\|_{\mathcal{X}} \leq C\left\|\Gamma^{0}\right\|_{\dot{\mathcal{B}}_{2,(\infty, 1)}^{\frac{1}{2}} \times H^{\frac{1}{2}} \times H^{\frac{1}{2}}} \leq C \kappa \delta \leq \frac{\delta}{2}
$$

for $\kappa$ small enough. On the other hand, in below we will prove that, if $\Gamma^{(1)}$ and $\Gamma^{(2)}$ belong to $B_{\delta}$, then under the assumptions of the claim, we have

$$
\left\|\Phi\left(\Gamma^{(1)}\right)-\Phi\left(\Gamma^{(2)}\right)\right\|_{\mathcal{X}} \leq \frac{1}{2}\left\|\Gamma^{(1)}-\Gamma^{(2)}\right\|_{\mathcal{X}} .
$$

Then estimates (3.43) and (3.44) easily yield the claim of proposition 3.1.

To prove (3.44), let $\Gamma^{(j)}:=\left(u_{j}, E_{j}, B_{j}\right)^{T} \in B_{\delta}$ for $j=1,2$. Write further

$$
e^{t \mathcal{A}} \Gamma_{\mathrm{err}}^{0}+\Gamma^{(j)}(t)=\left(\bar{u}_{j}, \bar{E}_{j}, \bar{B}_{j}\right)^{T}
$$

and set $\Gamma:=\Gamma^{(1)}-\Gamma^{(2)}:=(u, E, B)^{T}$, and $\Phi\left(\Gamma^{(j)}\right):=\tilde{\Gamma}^{(j)}=\left(\tilde{u}_{j}, \tilde{E}_{j}, \tilde{B}_{j}\right)^{T}$ be given by (3.1). Finally, let $\tilde{\Gamma}:=\tilde{\Gamma}^{(1)}-\tilde{\Gamma}^{(2)}:=(\tilde{u}, \tilde{E}, \tilde{B})^{T}$. 
We decompose $\tilde{u}$ into $\tilde{u}=\tilde{u}^{(a)}+\tilde{u}^{(b)}$, with $\tilde{u}^{(a)}$ accounting for the convection term

$$
\begin{aligned}
\tilde{u}^{(a)}: & =-\int_{0}^{t} e^{\left(t-t^{\prime}\right) \Delta} \mathbb{P} \nabla\left(u_{1} \otimes u+u \otimes u_{2}+u \otimes u_{\text {per }}+u_{\text {per }} \otimes u\right) d t^{\prime} \\
& +\int_{0}^{t} e^{\left(t-t^{\prime}\right) \Delta} \mathbb{P}\left(E_{1} \wedge B+E \wedge B_{2}+E \wedge B_{\text {per }}+E_{\text {per }} \wedge B\right) d t^{\prime} \\
& +\int_{0}^{t} e^{\left(t-t^{\prime}\right) \Delta} \mathbb{P}\left(\left(u \wedge B_{1}\right) \wedge B_{1}+\left(u_{2} \wedge E\right) \wedge B_{1}+\left(u_{2} \wedge B_{2}\right) \wedge B\right) d t^{\prime} \\
& +\int_{0}^{t} e^{\left(t-t^{\prime}\right) \Delta} \mathbb{P}\left(\left(u \wedge B_{\text {per }}\right) \wedge B_{\text {per }}\right) d t^{\prime}
\end{aligned}
$$

and

$$
\tilde{u}^{(b)}:=\int_{0}^{t} e^{\left(t-t^{\prime}\right) \Delta} \mathbb{P}\left(\left(u_{\mathrm{per}} \wedge B\right) \wedge B_{\mathrm{per}}+\left(u_{\mathrm{per}} \wedge B_{\mathrm{per}}\right) \wedge B\right) d t^{\prime}
$$

Moreover, the electro-magnetic field $(\tilde{E}, \tilde{B})$ satisfies

$$
\begin{aligned}
\partial_{t} \tilde{E}-\nabla \wedge \tilde{B}+\tilde{E} & =u_{1} \wedge B+u \wedge B_{2}+u \wedge B_{\text {per }}+u_{\text {per }} \wedge B \\
\partial_{t} \tilde{B}+\nabla \wedge \tilde{E} & =0
\end{aligned}
$$

with 0 initial data. Applying Lemmas 3.3 , 3.4 and 3.5 immediately gives estimates (3.43) and (3.44) and thus finishes the proof of the proposition 3.1 . 


\section{A Spectral properties of Maxwell's operator}

Here, we detail the linear analysis of Maxwell's system (3.13), whose spectral decomposition will be essential for the proof of Lemma 3.2 .

Clearly, Maxwell's system (3.13) may be recast as

$$
\partial_{t}\left(\begin{array}{l}
E \\
B
\end{array}\right)=\mathcal{L}\left(\begin{array}{l}
E \\
B
\end{array}\right)+\left(\begin{array}{c}
G \\
0
\end{array}\right)
$$

where Maxwell's operator $\mathcal{L}$ is given by

$$
\mathcal{L}:=\left(\begin{array}{cc}
-\mathrm{Id} & \nabla \wedge \\
-\nabla \wedge & 0
\end{array}\right) .
$$

More precisely, the operator

$$
\mathcal{L}: \mathcal{D}(\mathcal{L}) \subset X \rightarrow X
$$

is defined as an unbounded linear operator, where

$$
X:=\left\{(E, B) \in\left(L^{2}\left(\mathbb{R}^{3}\right)\right)^{2} \quad \text { such that } \operatorname{div} B=0\right\},
$$

whose domain is given by

$$
\mathcal{D}(\mathcal{L}):=\left\{(E, B) \in X \quad(\mathbb{P} E, B) \in\left(H^{1}\left(\mathbb{R}^{3}\right)\right)^{2}\right\},
$$

where $\mathbb{P}: L^{2}\left(\mathbb{R}^{3}\right) \rightarrow L^{2}\left(\mathbb{R}^{3}\right)$ denotes the Leray projector over solenoidal vector fields.

Next, in order to refine our understanding of the action of the semigroup and the ensuing behaviour of the electromagnetic field $(E, B)$, we conduct a spectral analysis of $\mathcal{L}$. Since, it has constant coefficients, we use the Fourier transform, which is denoted by

$$
\mathcal{F} f(\xi)=\hat{f}(\xi):=\int_{\mathbb{R}^{d}} e^{-i \xi \cdot x} f(x) d x
$$

and its inverse by

$$
\mathcal{F}^{-1} g(x)=\tilde{g}(x):=\frac{1}{(2 \pi)^{d}} \int_{\mathbb{R}^{d}} e^{i x \cdot \xi} g(\xi) d \xi .
$$

For every $\xi \in \mathbb{R}^{3} \backslash\{0\}$, we further define the subspace

$$
\mathcal{E}(\xi):=\left\{(e, b) \in \mathbb{C}^{3} \times \mathbb{C}^{3} \text { such that } \xi \cdot b=0\right\},
$$

and the linear finite-dimensional operator $\hat{\mathcal{L}}(\xi): \mathcal{E}(\xi) \rightarrow \mathcal{E}(\xi)$ by

$$
\hat{\mathcal{L}}(\xi)\left(\begin{array}{l}
e \\
b
\end{array}\right):=\left(\begin{array}{c}
-e+i \xi \wedge b \\
-i \xi \wedge e
\end{array}\right)
$$


Clearly, $\mathcal{E}(\xi)$ is a 5-dimensional vector subspace of $\mathbb{C}^{3} \times \mathbb{C}^{3}$ and any $(E, B) \in X$ satisfies that $(\hat{E}(\xi), \hat{B}(\xi)) \in \mathcal{E}(\xi)$, for almost every $\xi \in \mathbb{R}^{3}$. Finally, note that, for any $(E, B) \in X$,

$$
\mathcal{F}\left(\mathcal{L}\left(\begin{array}{l}
E \\
B
\end{array}\right)\right)(\xi)=\left(\begin{array}{c}
-\hat{E}+i \xi \wedge \hat{B} \\
-i \xi \wedge \hat{E}
\end{array}\right)=\hat{\mathcal{L}}(\xi)\left(\begin{array}{l}
\hat{E} \\
\hat{B}
\end{array}\right)
$$

and

$$
\mathcal{F}\left(e^{t \mathcal{L}}\left(\begin{array}{l}
E \\
B
\end{array}\right)\right)(\xi)=e^{t \hat{\mathcal{L}}(\xi)}\left(\begin{array}{l}
\hat{E} \\
\hat{B}
\end{array}\right)
$$

Then, we have the following properties proven in [1].

Proposition A.1. For $|\xi| \neq \frac{1}{2}$ the distinct eigenvalues of $\hat{\mathcal{L}}(\xi)$ are $\lambda_{0}=-1$, $\lambda_{+}(\xi)$ and $\lambda_{-}(\xi)$, with

$$
\lambda_{ \pm}(\xi)=\frac{-1 \pm \sqrt{1-4|\xi|^{2}}}{2} .
$$

Furthermore, there exists a basis of eigenvectors (i.e. $\hat{\mathcal{L}}(\xi)$ is diagonalizable) and the eigenspaces corresponding to $\lambda_{0}, \lambda_{+}(\xi)$ and $\lambda_{-}(\xi)$ are respectively given by

$$
\begin{aligned}
\mathcal{E}_{0}(\xi) & =\left\langle\left(\begin{array}{c}
\xi \\
0
\end{array}\right)\right\rangle, \\
\mathcal{E}_{+}(\xi) & =\left\{\left(\begin{array}{c}
e \\
\frac{-i}{\lambda_{+}} \xi \times e
\end{array}\right) \in \mathbb{C}^{3} \times \mathbb{C}^{3}: e \in \mathbb{C}^{3}, \xi \cdot e=0\right\} \\
& =\left\{\left(\begin{array}{c}
\frac{-i}{\lambda_{-}} \xi \times b \\
b
\end{array}\right) \in \mathbb{C}^{3} \times \mathbb{C}^{3}: b \in \mathbb{C}^{3}, \xi \cdot b=0,\right\} \\
\mathcal{E}_{-}(\xi) & =\left\{\left(\begin{array}{c}
e \\
\frac{-i}{\lambda_{-}} \xi \times e
\end{array}\right) \in \mathbb{C}^{3} \times \mathbb{C}^{3}: e \in \mathbb{C}^{3}, \xi \cdot e=0\right\} \\
& =\left\{\left(\begin{array}{c}
\frac{-i}{\lambda_{+}} \xi \times b \\
b
\end{array}\right) \in \mathbb{C}^{3} \times \mathbb{C}^{3}: b \in \mathbb{C}^{3}, \xi \cdot b=0\right\} .
\end{aligned}
$$

For any $\xi \in \mathbb{R}^{3} \backslash\{0\}$, such that $|\xi|=\frac{1}{2}$, the distinct eigenvalues of $\mathcal{L}(\xi)$ are $\lambda_{0}=-1$ and $\lambda_{1}=-\frac{1}{2}$. Furthermore, $\hat{\mathcal{L}}(\xi)$ is not diagonalizable and the eigenspaces corresponding to $\lambda_{0}$ and $\lambda_{1}$ are respectively given by

$$
\begin{aligned}
\mathcal{E}_{0}(\xi) & =\left\langle\left(\begin{array}{c}
\xi \\
0
\end{array}\right)\right\rangle, \\
\mathcal{E}_{1}(\xi) & \left.=\left\{\left(\begin{array}{c}
e \\
\frac{2 i}{\sigma c} \xi \times e
\end{array}\right) \in \mathbb{C}^{3} \times \mathbb{C}^{3}: e \in \mathbb{C}^{3}, \xi \cdot e=0 \text { (and } e_{3}=0 \text { if } d=2\right)\right\} \\
& \left.=\left\{\left(\begin{array}{c}
\frac{2 i}{\sigma c} \xi \times b \\
b
\end{array}\right) \in \mathbb{C}^{3} \times \mathbb{C}^{3}: b \in \mathbb{C}^{3}, \xi \cdot b=0 \text { (and } b_{1}=b_{2}=0 \text { if } d=2\right)\right\} .
\end{aligned}
$$


The generalized eigenspace corresponding to $\lambda_{1}$ is given by

$\mathcal{K}_{1}(\xi)=\left\{\left(\begin{array}{l}e \\ b\end{array}\right) \in \mathbb{C}^{3} \times \mathbb{C}^{3}: \xi \cdot e=\xi \cdot b=0\right.$ (and $e_{3}=b_{1}=b_{2}=0$ if $\left.\left.d=2\right)\right\}$.

Remark A.1. One easily verifies that the operator $\hat{\mathcal{L}}(\xi)$ is not normal (i.e. it does not commute with its adjoint) and, therefore, the eigenspaces $\mathcal{E}_{0}(\xi)$, $\mathcal{E}_{+}(\xi)$ and $\mathcal{E}_{-}(\xi)$ are not all orthogonal to each other. However, it is seen that each $\mathcal{E}_{ \pm}(\xi)$ is orthogonal to $\mathcal{E}_{0}(\xi)$.

Lemma A.1. Let $\xi \in \mathbb{R}^{3}$ and consider the eigenvalues $\lambda_{ \pm}(\xi)$ defined by (A.1). Then, if $|\xi| \leq \frac{1}{2}$,

$$
\begin{aligned}
& -1 \leq \lambda_{-}(\xi) \leq-\frac{1}{2} \leq-|\xi| \leq-2|\xi|^{2} \leq \lambda_{+}(\xi) \leq-|\xi|^{2}, \\
& \text { and } \quad \sqrt{1-(2|\xi|)^{2}} \leq \frac{\lambda_{-}(\xi)-\lambda_{+}(\xi)}{\lambda_{-}(\xi)} \leq 2 \sqrt{1-(2|\xi|)^{2}},
\end{aligned}
$$

and, if $|\xi| \geq \frac{1}{2}$

$$
\begin{gathered}
\Re\left(\lambda_{-}(\xi)\right)=\Re\left(\lambda_{+}(\xi)\right)=-\frac{1}{2}, \quad\left|\lambda_{+}(\xi)\right|=\left|\lambda_{-}(\xi)\right|=|\xi|, \\
\text { and } \quad\left|\frac{\lambda_{-}(\xi)-\lambda_{+}(\xi)}{\lambda_{-}(\xi)}\right|=2 \sqrt{1-\left(\frac{1}{2|\xi|}\right)^{2}} .
\end{gathered}
$$

Thanks to Proposition A.1, decomposing the initial data $\left(E^{0 c}, B^{0 c}\right) \in X$ and the source terms $G^{c} \in L_{\text {loc }}^{1}\left(\mathbb{R}^{+} ; L^{2}\left(\mathbb{R}^{3}\right)\right)$ using the eigenspaces of $\hat{\mathcal{L}}(\xi)$, we write for almost every $\xi \in \mathbb{R}^{3}$,

$$
\left(\begin{array}{c}
\hat{E}^{0} \\
\hat{B}^{0}
\end{array}\right)=\left(\begin{array}{c}
\frac{\xi \cdot \hat{E}^{0}}{|\xi|^{2}} \xi \\
0
\end{array}\right)+\left(\begin{array}{c}
e^{0} \\
\frac{-i}{\lambda_{-}} \xi \times e^{0}
\end{array}\right)+\left(\begin{array}{c}
\frac{-i}{\lambda_{-}} \xi \times b^{0} \\
b^{0}
\end{array}\right),
$$

where $\xi \cdot e^{0}=\xi \cdot b^{0}=0$, and

$$
\left(\begin{array}{c}
\hat{G} \\
0
\end{array}\right)=\left(\begin{array}{c}
\frac{\xi \cdot \hat{G}}{|\xi|^{2}} \xi \\
0
\end{array}\right)+\left(\begin{array}{c}
e \\
\frac{-i}{\lambda_{-}} \xi \times e
\end{array}\right)+\left(\begin{array}{c}
\frac{-i}{\lambda_{-}} \xi \times b \\
b
\end{array}\right)
$$

where $\xi \cdot e=\xi \cdot b=0$.

Next, in view of Proposition A.1, the semigroup $e^{t \hat{\mathcal{L}}}$ acts on (3.21) as

$$
e^{t \hat{\mathcal{L}}}\left(\begin{array}{c}
\hat{E}^{0} \\
\hat{B}^{0}
\end{array}\right)=e^{-t}\left(\begin{array}{c}
\xi \cdot \hat{E} \\
|\xi|^{2} \\
0
\end{array}\right)+e^{t \lambda_{-}}\left(\begin{array}{c}
e^{0} \\
\frac{-i}{\lambda_{-}} \xi \times e^{0}
\end{array}\right)+e^{t \lambda_{+}}\left(\begin{array}{c}
\frac{-i}{\lambda_{-}} \xi \times b^{0} \\
b^{0}
\end{array}\right)
$$


and on $\underline{A .3}$ as

$$
\begin{aligned}
\int_{0}^{t} e^{(t-\tau) \hat{\mathcal{L}}}\left(\begin{array}{c}
\hat{G} \\
0
\end{array}\right)(\tau) d \tau & =\int_{0}^{t} e^{-(t-\tau)}\left(\begin{array}{c}
\frac{\xi \cdot \hat{G}}{|\xi|^{2}} \xi \\
0
\end{array}\right) d \tau \\
& +\int_{0}^{t} e^{(t-\tau) \lambda_{-}}\left(\begin{array}{c}
e \\
\frac{-i}{\lambda_{-}} \xi \times e
\end{array}\right) d \tau \\
& +\int_{0}^{t} e^{(t-\tau) \lambda_{+}}\left(\begin{array}{c}
\frac{-i}{\lambda_{-}} \xi \times b \\
b
\end{array}\right) d \tau .
\end{aligned}
$$

Therefore, Duhamel's formula (A.2) yields that

$$
\hat{B}(t)=\frac{-i c}{\lambda_{-}} e^{t \lambda_{-}} \xi \times e^{0}+e^{t \lambda_{+}} b^{0}+\int_{0}^{t}\left(\frac{-i}{\lambda_{-}} e^{(t-\tau) \lambda_{-}} \xi \times e+e^{(t-\tau) \lambda_{+}} b\right) d \tau .
$$

Further substituting

$$
\begin{aligned}
\frac{i c}{\lambda_{-}} \xi \times e^{0} & =b^{0}-\hat{B}^{0}, \\
b & =\frac{i}{\lambda_{-}} \xi \times e,
\end{aligned}
$$

which is deduced from the second components of (3.21) and (A.3), we obtain

$$
\hat{B}(t)=e^{t \lambda_{-}} \hat{B}^{0}+\left(e^{t \lambda_{+}}-e^{t \lambda_{-}}\right) b^{0}+\frac{i}{\lambda_{-}} \int_{0}^{t}\left(e^{(t-\tau) \lambda_{+}}-e^{(t-\tau) \lambda_{-}}\right) \xi \times e d \tau .
$$

\section{References}

[1] D. Arsénio, S. Ibrahim and S. Masmoudi. A derivation of the magnetohydrodynamic system from Navier-Stokes-Maxwell systems. Arch. Ration. Mech. Anal., 216, no. 3, 767-812, 2015.

[2] H. Bahouri, J.-Y. Chemin, and R. Danchin. Fourier Analysis and Nonlinear Partial Differential Equations, Grundlehren Math. Wiss. 343, Springer-Verlag, Heidelberg, 2011.

[3] D. Biskamp. Nonlinear magnetohydrodynamics, volume 1 of Cambridge Monographs on Plasma Physics. Cambridge University Press, Cambridge, 1993.

[4] J.-M. Bony. Calcul symbolique et propagation des singularités pour les équations aux dérivées partielles non linéaires. Ann. Sci. École Norm. Sup. (4), 14(2):209-246, 1981.

[5] M. Cannone, Q. Chen, and C. Miao. A losing estimate for the ideal MHD equations with application to blow-up criterion. SIAM J. Math. Anal., 38(6):1847-1859 (electronic), 2007. 
[6] J. -Y. Chemin, Perfect incompressible Fluids, Clarendon press, Oxford, 1998.

[7] J. -Y. Chemin and I. Gallagher. On the global well-posedness of the 3-D Navier-Stokes equations with large initial data. Ann. Sci. cole Norm. Sup. 39(4), no. 4, 679698, 2006.

[8] J.-Y. Chemin and N. Lerner. Flot de champs de vecteurs non lipschitziens et équations de Navier-Stokes. J. Differential Equations, 121(2):314-328, 1995.

[9] P. A. Davidson. An introduction to magnetohydrodynamics. Cambridge Texts in Applied Mathematics. Cambridge University Press, Cambridge, 2001.

[10] H. Fujita and T. Kato. On the Navier-Stokes initial value problem I, Archiv for Rationnal Mechanic Analysis, 16, (1964), pages 269315.

[11] P. Germain, S. Ibrahim and S. Masmoudi. Well-posedness of the NavierStokes-Maxwell equations, Proc. Royal Soc. Edinburgh, 144 A, 71-86, 2014 .

[12] H-J Ko and G. S. Dulikravich. A fully non-linear theory of electromagnetic-hydrodynamics, Int. Jour. of Non-Linear Mech., 35, (2000) 709-719.

[13] M. Kyed. Time-periodic solutions to the Navier-Stokes Equations. Habilitationsschrift. Technische Universtit at Darmstads, 2012.

[14] S. Ibrahim and T. Yoneda. Local solvability and loss of smoothness of the Navier-Stokes-Maxwell equations with large initial data, Jour. Math. Anal. Appl, 396, 2012.

[15] S. Ibrahim and S. Keraani. Global small solutions of the Navier-StokesMaxwell equations, SIAM jour. Math. Anal., 43 (2011), 2275-2295.

[16] P. G. Lemarié-Rieusset. Recent developments in the Navier-stokes problem. CRC Press, (2002).

[17] J. Leray. Essai sur le mouvement dun liquide visqueux remplissant l'espace, Acta Mathematica, 63, (1933), pages 193-248, 555-561.

[18] N. Masmoudi. Global well-posedness for the Maxwell-Navier-Stokes system in 2D. Journal de Math. Pures et Appl. 93 (2010) 559571. 